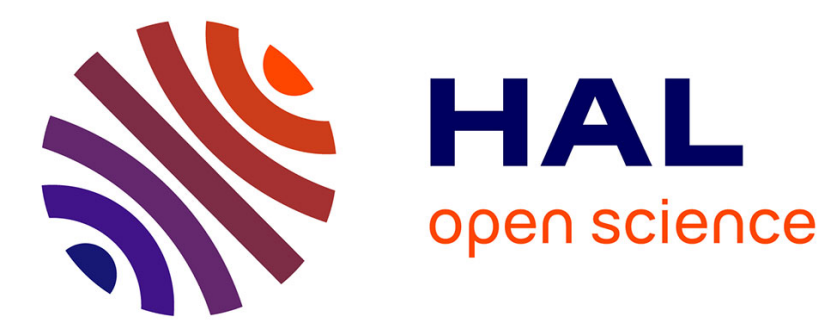

\title{
Phenomenological multi-mechanisms constitutive modelling for thermoplastic polymers, implicit implementation and experimental validation
}

Francis Praud, Georges Chatzigeorgiou, Jérôme Bikard, Fodil Meraghni

\section{To cite this version:}

Francis Praud, Georges Chatzigeorgiou, Jérôme Bikard, Fodil Meraghni. Phenomenological multimechanisms constitutive modelling for thermoplastic polymers, implicit implementation and experimental validation. Mechanics of Materials, 2017, 114, pp.9-29. 10.1016/j.mechmat.2017.07.001 . hal-01575686

\author{
HAL Id: hal-01575686 \\ https://hal.science/hal-01575686
}

Submitted on 21 Aug 2017

HAL is a multi-disciplinary open access archive for the deposit and dissemination of scientific research documents, whether they are published or not. The documents may come from teaching and research institutions in France or abroad, or from public or private research centers.
L'archive ouverte pluridisciplinaire $\mathbf{H A L}$, est destinée au dépôt et à la diffusion de documents scientifiques de niveau recherche, publiés ou non, émanant des établissements d'enseignement et de recherche français ou étrangers, des laboratoires publics ou privés. 


\title{
Phenomenological multi-mechanisms constitutive modelling for thermoplastic polymers, implicit implementation and experimental validation
}

\author{
F. Praud ${ }^{\mathrm{a}, \mathrm{b}}$, G. Chatzigeorgiou ${ }^{\mathrm{a}}$, J. Bikard $^{\mathrm{c}}$, F. Meraghnia ${ }^{\mathrm{a}, *}$ \\ ${ }^{a}$ Arts et Metiers ParisTech, LEM3-UMR 7239 CNRS, 4 rue Augustin Fresnel, 57078 \\ Metz, France \\ ${ }^{b}$ IRT Jules Verne, Chemin du Chaffault, 44340 Bougenais, France \\ ${ }^{c}$ Solvay, 85 rue des Freres Perret, BP 62, 69192 Saint Fons Cedex, France
}

\begin{abstract}
In this work, a phenomenological model for thermoplastic polymers involving several mechanisms is proposed. The constitutive equations lie within the framework of thermodynamics and account for both viscoelasticty, viscoplasticity and ductile damage. An implicit numerical scheme utilizing the "return mapping algorithm" is provided along with the formulation of the tangent operator. The parameters of the developed model are experimentally identified through a gradient-based inverse method using three strain-controlled configurations. The model validation is achieved by comparing numerical results with experimental data obtained on a cyclic loading configuration test. Finally, the capabilities of the proposed model are demonstrated with a series of numerical simulations where complex cyclic and non-proportional loading conditions are applied as well as with a structural FE application.
\end{abstract}

Keywords: Constitutive behaviour; Viscoelasticity; Viscoplasticity; Ductile

\footnotetext{
*Corresponding author. Tel: +33(0)387375459, Fax :+33(0)387374284

Email address: fodil.meraghni@ensam.eu (F. Meraghni)
} 
damage; Implicit implementation; Polymers.

\section{Introduction}

Thermoplastics and more especially semi-crystalline polymers are well known to exhibit a mechanical rate-dependent behaviour combining both solid and fluid properties. They also appear to be sensitive to the environmental conditions, namely relative humidity and temperature (Launay et al., 2013c; Arif et al., 2014a). Investigations showed that these materials are also subjected to stiffness reduction during loading caused by the existence of damage mechanisms related to the initiation, the growth and the coalescence of micro defects (Detrez et al., 2011).

The growing interest for this type of materials, especially for automotive applications, has contributed to many modelling efforts aiming at capturing the complex behaviour of thermoplastics or thermoplastic based composites. Among the existing models, those based on phenomenological approaches account for one or several dissipative mechanisms. Indeed, to capture the overall behaviour of polymers or polymeric composites, some authors refer to the theories of viscoelasticity (Moreau et al., 2005; Khan et al., 2006), viscoplasticity (Achour et al., 2015; Drosdov and Christiansen, 2007), cou-

pled viscoelasticity and viscoplasticity (Miled et al., 2011), damage (Nouri et al., 2009) or even both coupled viscoelsticity, viscoplasticity and damage (Launay et al., 2011, 2013a; Krairi and Doghri, 2014; Lawrimore II et al., 2016). It is worth noticing that some other models integrate aspects related to physics of polymers, such as the molecular chains network reorganization 
(Maurel-Pantel et al., 2015) or the partial phase transformation between crystalline and amorphous phases (Oshmyan et al., 2005). Other works (Arruda et al., 1995) have introduced conformational rearrangement as mechanisms behind yielding and plasticity of glassy polymers. More recent studies (Voyiadjis and Samadi-Dooki, 2016) consider the deformation of glassy polymers through the evolution of the free volume and shear transformation zones during the generation of plastic strain. Despite their efficiency, the latter type of constitutive models requires physical data that can be difficult to measure experimentally or to control during the manufacturing process. Thermodynamically derived phenomenological models are more suitable since they require material parameters that can be identified from conventional mechanical tests. Indeed, the phenomenological approaches focus only on the overall description of the material behaviour, using internal state variables. For polymers, elementary mechanisms such as anelasticity or damage are rate dependent and are introduced through permanent strain and stiffness reduction, along with their related dissipation. When several phenomena occur simultaneously, these elementary dissipative mechanisms can be modelled together using coupled formulations.

With regard to viscoelaticity, two main modelling approaches can be used, namely, the hereditary integral (Miled et al., 2011; Krairi and Doghri, 2014) and the differential form. The hereditary integral representation is based on the concept of relaxation or creep functions that need to be integrated through the whole time history to establish the stress-strain relationship. The main interest of the integral form is that the viscoelastic mechanism can 
be modelled though a single strain state variable. However, its practical use is generally adopted for the case of isotropic viscoelasticity and an extension to non-isotropic media is not straightforward. In addition, the fully coupled thermomechanical behaviour cannot be easily integrated within the integral formalism, since such coupling effects require derivative operations of the thermodynamic potentials that may be complicated within a formulation involving time integral. On the other hand, the differential representation is based on the assembly of rheological elements that store or dissipate energy, namely: springs or dash-pots respectively. Such a description may involve a certain number of strain state variables in order to capture properly the complete viscoelastic response. The main advantage of this formalism is that it permits an explicit splitting of the various energetic terms on the thermodynamic potentials. It also offers more flexibilities for the integration of additional effects such as thermally activated mechanisms (Yu et al., $2017 \mathrm{a}, \mathrm{b})$ or anisotropy.

Concerning damage, several approaches can be found in the literature. In some of them, the effect of damage is introduced through a micromechanical representation based on a physical definition of a damaged representative volume element that accounts for material micro-discontinuities (Gurson, 1977). For semi-crystalline polymers such a description requires a deep knowledge of the involved degradation mechanisms at the macromolecular chains scale (Detrez et al., 2011) and also a proper modelling theory. This task is neither straightforward nor feasible due to the fact that the definition of a continuum medium does not stand at that scale. Alternatively, damage can also 
be taken into account in a purely phenomenological manner, where only the effect of the damage consequences on the overall material response, namely: the stiffness reduction. Such effect is then introduced in the framework of thermodynamics with the help of one or several internal state variables along with the appropriate evolution laws (Lemaitre and Chaboche, 1990; Lemaitre and Desmorat, 2005). The damage is hence considered as a continuum quantity describing the state of the material at the scale of interest.

This paper proposes a model for polymers accounting for viscoelasticity, viscoplasticity and ductile damage. It is formulated within a proper thermodynamical framework and is applied to predict the deformation of semi-crystalline polymers under the small strain assumption. It is well established that such materials are rate and temperature dependent (Arruda et al., 1995). The temperature may play a significant role due to the selfheating phenomenon arising from dissipative mechanisms, especially upon cyclic loading (Yu et al., 2017b). In the present paper, only the rate effect is accounted for, while the material is assumed to stay under isothermal conditions. The developed constitutive model presents several similar features with the one proposed by Krairi and Doghri (2014), mainly on the integration of viscoplasticity and damage, but it has certain important differences:

- Here the differential formulation is adopted for the viscoelastic part, which is described with the help of a series of Kelvin-Voigt branches. Krairi and Doghri (2014) utilized an integral formulation and the viscoelastic properties are expressed through Prony series. It should be mentioned that viscoelastic models containing several Kelvin-Voigt 
branches in series or several Maxwell branches in parallel (Prony series) are both equivalent in term of global response, including both creep and relaxation effects. Their practical difference is the following: Prony series imply an additive decomposition of the stress as proposed by several authors, among them (Miled et al., 2011; Krairi and Doghri, 2014). Indeed, the sum of the stresses in each single Maxwell branch provides the total stress, while all the Maxwell branches share the same strain. On the other hand, a multiple Kelvin Voigt implies an additive decomposition of the strain. Each Kelvin Voigt branch has its own viscoelastic strain, while all branches share the same stress. The second formalism appears more straightforward in a strain driven approach like the "return mapping algorithm".

- The elastic and viscoelastic formulation, both in the theoretical and the numerical implementation section (Section 3), are structured in a general context that permits to account for anisotropic response. The formalism described by Krairi and Doghri (2014) considers only isotropic viscoelastic response.

- The proposed time implicit numerical scheme includes the definition of a tangent operator even in anisotropic cases, that is essential for the finite element calculations.

- The parameter identification strategy accounts for the strong couplings between viscoelastic, viscoplastic and damage mechanisms. Krairi and Doghri (2014) identified the viscoelastic response by using Dynamic Mechanical Analysis (DMA) tests. Such tests are performed in low 
stress levels and may not account properly for the material viscoelastic response at the desired (usually moderate and high) stress conditions. To resolve this issue, the identification strategy described in Section 3 utilizes experimental results closer to the conditions of actual applications and involving the expected coupling between viscoelasticity, viscoplasticity and ductile damage.

An important task of this work is also to verify that the numerical implementation of the proposed model captures the material response under non-proportional and complex loading conditions (see for instance the structural example of Section 6). Such verification is essential to validate the 3D numerical implementation of the model as well as its applicability for studying the behaviour of thermoplastic based composites through multiscale micromechanical schemes, whether by mean field theories (Lagoudas et al., 1991; Chaboche et al., 2005; Miled et al., 2013) or periodic homogenization (Chatzigeorgiou et al., 2015, 2016).

This paper is structured as follows: In the second section, the constitutive equations and the thermodynamics framework of the proposed model are presented. The third section is devoted to the time implicit numerical implementation, suitable for the computation of the tangent operator. A user material algorithm has been formulated and implemented into the finite element code ABAQUS. The fourth section focuses on the experimental procedure and the identification strategy of the model parameters for the polyamide $(6,6)$, as well as its experimental validation under several loading conditions and strain rates. The fifth section introduces examples of uni-axial 
simulations where the material is subjected to cyclic and non-proportional loadings. The computed results allow to highlight the dissipative behaviour of the material by examining the evolution of the state variables during loading. Energy balances are also performed in order to assess the part of the strain energy that is dissipated and the part that is stored in the material. The sixth section is devoted to the application of the proposed model for FE analysis through an example of 3D structure subjected to a complex cyclic loading. The last section summarizes the main conclusions and discuses the perspectives related to this work.

The following notation is adopted in this work: bold and blackboard symbols respectively denote second and fourth order tensors while other symbols are scalar quantities. The twice contracted and dyadic products are given by:

$$
\boldsymbol{A}: \boldsymbol{B}=A_{i j} B_{i j}, \quad(\mathbb{A}: \boldsymbol{B})_{i j}=A_{i j k l} B_{k l}, \quad(\boldsymbol{A} \otimes \boldsymbol{B})_{i j k l}=A_{i j} B_{k l} .
$$

Moreover, all the second order tensors are symmetric $\left(A_{i j}=A_{j i}\right)$ and all the fourth order tensors have at least the minor symmetries $\left(A_{i j k l}=A_{j i k l}=\right.$ $\left.A_{i j l k}\right)$. Consequently, they can be respectively reduced to $6 \times 1$ and $6 \times 6$ matrices according to the Voigt notation. $I$ and $\mathbb{I}$ are the second and the fourth order identity tensors, respectively, defined as:

$$
(\boldsymbol{I})_{i j}=\delta_{i j}, \quad(\mathbb{I})_{i j k l}=\frac{1}{2}\left(\delta_{i k} \delta_{j l}+\delta_{i l} \delta_{j k}\right),
$$

where $\delta_{i j}$ is the Kronecker symbol. The inverse of a fourth order tensor $\mathbb{A}$, that has the minor symmetries, is the fourth order tensor $\mathbb{A}^{-1}$ for which $\mathbb{A}: \mathbb{A}^{-1}=\mathbb{A}^{-1}: \mathbb{A}=\mathbb{I}$. The operators $h y d(\boldsymbol{\sigma})$ and $\boldsymbol{\operatorname { d e v }}(\boldsymbol{\sigma})$ designate the 
hydrostatic pressure and the deviatoric part of a stress tensor $\boldsymbol{\sigma}$ respectively, whereas $e q(\boldsymbol{\sigma})$ is the equivalent Von Mises stress:

$$
\begin{gathered}
h y d(\boldsymbol{\sigma})=\frac{1}{3} \operatorname{tr}(\boldsymbol{\sigma}) \\
\boldsymbol{d e} \boldsymbol{v}(\boldsymbol{\sigma})=\boldsymbol{\sigma}-h y d(\boldsymbol{\sigma}) \boldsymbol{I} \\
e q(\boldsymbol{\sigma})=\sqrt{\frac{3}{2}(\boldsymbol{d e} \boldsymbol{v}(\boldsymbol{\sigma}): \boldsymbol{d e} \boldsymbol{v}(\boldsymbol{\sigma}))}
\end{gathered}
$$

\section{Constitutive model and thermodynamical framework}

The objective of this section is to present the formulation of a thermodynamically based phenomenological model accounting for viscoelasticity, viscoplasticity and ductile damage in semi-crystalline polymers. The proposed model is described by the rheological scheme given in Figure 1a. It is composed of several elements positioned in series which are detailed below. The damage is introduced through the continuum damage theory based on the well-known principle of effective stress (Lemaitre and Chaboche, 1990). First introduced for the uni-axial case, a similar formalism can be easily applied in 3D if damage is regarded as an isotropic phenomenon. Then, the definition of the effective stress tensor, that has been adopted here, is simply given by:

$$
\widetilde{\boldsymbol{\sigma}}=\frac{\boldsymbol{\sigma}}{1-D}
$$

Where $D$ is the damage variable expressed as a scalar quantity. Actually, even if the material is assumed to be initially isotropic, the overall response may exhibit anisotropic effects induced by damage that are governed by the principal stress directions. Example of extensions to 3D damage induced anisotropy can be found in the literature (Lemaitre and Desmorat, 2005). 
Nevertheless such a description requires quantification through further experimental data highlighting the behaviour in the other directions or from experiments where the material is subjected to multi-axial loading conditions. In the absence of these data, the simplest assumption remains the case of isotropic damage.

The rheological model consists of the following elements:

- One single linear spring subjected to an elastic strain $\varepsilon_{e}$.

- $N$ viscoelastic Kelvin-Voigt branches, consisting of a linear spring and a linear dash-pot assembled in parallel (Figure 1b). Each Kelvin-Voigt branch " $i$ " is subjected to a viscoelastic strain $\boldsymbol{\varepsilon}_{\boldsymbol{v}_{i}}$. From a conceptual point of view, a single linear Kelvin-Voigt offers interesting capabilities in capturing the creep and relaxation effects but only with one characteristic time and consequently within a limited range of loading frequency/rate. Considering several Kelvin-Voigt branches allow to account for similar effects within an extended range of loading frequency/rate (Ottosen and Ristinmaa, 2005).

- One viscoplastic branch, consisting of a frictional element, a non-linear spring and a non-linear dash-pot both assembled in parallel (Figure 1c). This branch is subjected to a viscoplastic strain $\varepsilon_{p}$ that is only generated if the equivalent effective stress $e q(\tilde{\boldsymbol{\sigma}})$ exceeds a certain threshold.

The assembly in series of all those elements implies an additive decomposition of the total strain $\varepsilon$ :

$$
\boldsymbol{\varepsilon}=\varepsilon_{\boldsymbol{e}}+\sum_{i=1}^{N} \boldsymbol{\varepsilon}_{\boldsymbol{v}_{i}}+\boldsymbol{\varepsilon}_{\boldsymbol{p}}
$$


while all the branches are acting to the same effective stress $\widetilde{\boldsymbol{\sigma}}$. Nevertheless, the viscoplastic branch is assumed to be only sensitive to deviatoric part of $\widetilde{\boldsymbol{\sigma}}$ through the effective equivalent stress $e q(\widetilde{\boldsymbol{\sigma}})$. The duality between stress and strain implies the existence of an effective equivalent viscoplastic strain denoted by the scalar variable $r$ that is connected to the viscoplactic strain tensor $\varepsilon_{p}$ through the evolution laws. It is worth noticing that, in the proposed model, the damage is assumed to be "ductile" as it only evolves when the viscoplastic strain is being generated, making those two mechanisms directly coupled (Lemaitre, 1985; Lemaitre and Desmorat, 2005; Krairi and Doghri, 2014).

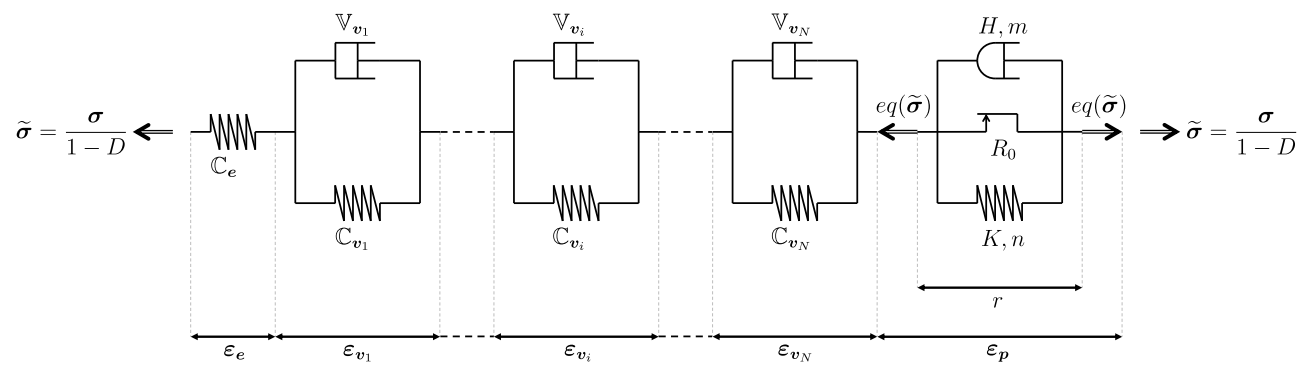

(a) Complete rheological scheme

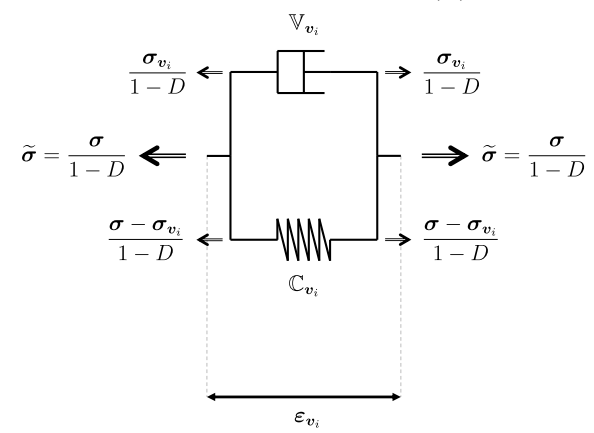

(b) Single Kelvin-Voigt branch

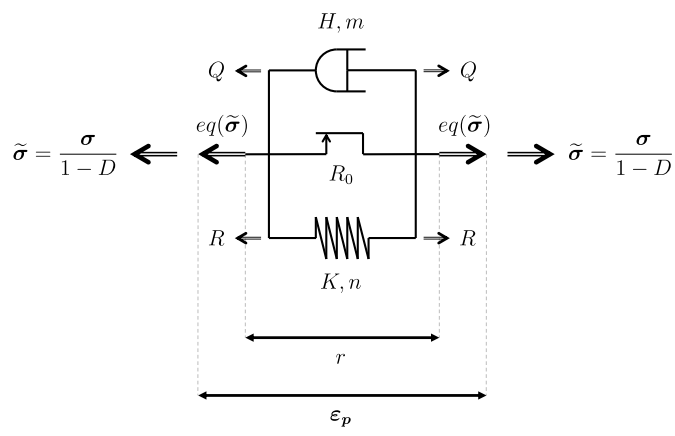

(c) Viscoplastic branch

Figure 1: Rheological scheme of the proposed model.

Finally, the observable state variable of the proposed model is $\varepsilon$ while 
the internal state variables are $\varepsilon_{\boldsymbol{v}_{i}}, \boldsymbol{\varepsilon}_{\boldsymbol{p}}, r$ and $D$. The elastic strain $\varepsilon_{\boldsymbol{e}}$ is not considered as a state variable but, for convenience, is expressed by the difference between the total strain and both the viscoelastic and viscoplastic strains.

\subsection{State laws}

The proposed model is formulated within the framework of thermodynamics (Lemaitre and Chaboche, 1990). The material state laws are based on a state potential, the Helmholtz free energy in the present case, that depends on the states variables previously mentioned. This potential is written as the sum of the stored energy functions of the single spring $\psi_{e}$, each Kelvin-Voigt branch $\psi_{v_{i}}$ and the viscoplastic branch $\psi_{p}$ :

$$
\psi\left(\boldsymbol{\varepsilon}, \boldsymbol{\varepsilon}_{\boldsymbol{v}_{i}}, \boldsymbol{\varepsilon}_{\boldsymbol{p}}, r, D\right)=\psi_{e}\left(\boldsymbol{\varepsilon}, \boldsymbol{\varepsilon}_{\boldsymbol{v}_{i}}, \boldsymbol{\varepsilon}_{\boldsymbol{p}}, D\right)+\sum_{i=1}^{N} \psi_{v_{i}}\left(\boldsymbol{\varepsilon}_{\boldsymbol{v}_{i}}, D\right)+\psi_{p}(r)
$$

with

$$
\begin{gathered}
\rho \psi_{e}=\frac{1}{2}\left(\boldsymbol{\varepsilon}-\sum_{i=1}^{N} \boldsymbol{\varepsilon}_{\boldsymbol{v}_{i}}-\boldsymbol{\varepsilon}_{\boldsymbol{p}}\right):(1-D) \mathbb{C}_{\boldsymbol{e}}:\left(\boldsymbol{\varepsilon}-\sum_{i=1}^{N} \boldsymbol{\varepsilon}_{\boldsymbol{v}_{i}}-\boldsymbol{\varepsilon}_{\boldsymbol{p}}\right), \\
\rho \psi_{v_{i}}=\frac{1}{2} \boldsymbol{\varepsilon}_{\boldsymbol{v}_{i}}:(1-D) \mathbb{C}_{\boldsymbol{v}_{i}}: \boldsymbol{\varepsilon}_{\boldsymbol{v}_{i}}, \\
\rho \psi_{p}=\int_{0}^{r} R(\xi) d \xi .
\end{gathered}
$$

Additionally, the associated variables $\boldsymbol{\sigma},-\boldsymbol{\sigma}_{\boldsymbol{v}_{i}}, \boldsymbol{- \sigma}, R$ and $-Y$ can be defined, and they are respectively given by differentiation of the potential with respect to the state variables $\boldsymbol{\varepsilon}, \boldsymbol{\varepsilon}_{\boldsymbol{v}_{i}}, \boldsymbol{\varepsilon}_{\boldsymbol{p}}, r$ and $D$ :

$$
\boldsymbol{\sigma}=\rho \frac{\partial \psi}{\partial \varepsilon}=(1-D) \mathbb{C}_{\boldsymbol{e}}:\left(\boldsymbol{\varepsilon}-\sum_{i=1}^{N} \boldsymbol{\varepsilon}_{\boldsymbol{v}_{i}}-\boldsymbol{\varepsilon}_{\boldsymbol{p}}\right)
$$




$$
\begin{gathered}
-\boldsymbol{\sigma}_{\boldsymbol{v}_{i}}=\rho \frac{\partial \psi}{\partial \boldsymbol{\varepsilon}_{\boldsymbol{v}_{i}}}=(1-D) \mathbb{C}_{\boldsymbol{v}_{i}}: \boldsymbol{\varepsilon}_{\boldsymbol{v}_{i}}-\boldsymbol{\sigma} \\
-\boldsymbol{\sigma}=\rho \frac{\partial \psi}{\partial \boldsymbol{\varepsilon}_{\boldsymbol{p}}} \\
R(r)=\rho \frac{\partial \psi}{\partial r} \\
-Y=\rho \frac{\partial \psi}{\partial D}=-Y_{e}-\sum_{i=1}^{N} Y_{v_{i}} .
\end{gathered}
$$

$\mathbb{C}_{\boldsymbol{e}}$ and $\mathbb{C}_{\boldsymbol{v}_{i}}$ denote the initial fourth order stiffness tensors of the single spring and the spring of the $i^{\text {th }}$ Kelvin-Voigt branch, respectively. These tensors are classically formulated for bulk isotropic materials and are defined by the Young modulus $E_{e}$ or $E_{v_{i}}$, respectively, as well as the Poisson ratio $\nu$ that is assumed to be the same in each stiffness tensor. The viscous stress $\boldsymbol{\sigma}_{\boldsymbol{v}_{i}}$ and the difference between $\boldsymbol{\sigma}$ and $\boldsymbol{\sigma}_{\boldsymbol{v}_{i}}$ respectively represent the stress acting on the linear dash-pot and the spring of the $i_{t h}$ Kelvin-Voigt branch (Figure 1b). The hardening function $R(r)$ stands for the effective equivalent stress acting on the non-linear spring of the viscoplastic branch (Figure 1c). $R(r)$ must be an increasing function defined positive and null at $r=0$. In this model, $R(r)$ is chosen under the form of a power law:

$$
R(r)=K r^{n}
$$

where $K$ and $n$ are material parameters. The total energy density release $Y$ is written as the sum of the energy density releases associated to the single spring and each Kelvin-Voigt branch:

$$
-Y_{e}=\rho \frac{\partial \psi_{e}}{\partial D}, \quad-Y_{v_{i}}=\rho \frac{\partial \psi_{v_{i}}}{\partial D} .
$$


$Y_{e}$ and $Y_{v_{i}}$ can be expressed either in term of strain or stress (Appendix A). The state and associated variables of the proposed model are summarised in Table 1.

Table 1: State and associated variables.

\begin{tabular}{|c|c|c|}
\hline \multicolumn{2}{|c|}{ State variables } & \multirow{2}{*}{$\begin{array}{c}\text { Associated viariables } \\
-\end{array}$} \\
\hline Observable & internal & \\
\hline \multirow[t]{5}{*}{$\varepsilon$} & & $\sigma$ \\
\hline & $\varepsilon_{\boldsymbol{v}_{i}}$ & $-\sigma_{v_{i}}$ \\
\hline & $\varepsilon_{p}$ & $-\sigma$ \\
\hline & $r$ & $R$ \\
\hline & $D$ & $-Y$ \\
\hline
\end{tabular}

\subsection{Evolution laws}

Under the assumption that there is no coupling between the thermal and mechanical dissipation, the second principle of thermodynamics implies the mechanical dissipation to be always positive or null through the Clausius Duhem inequality, where the rate of the dissipated energy $\Phi$ is expressed by the difference between the rate of the strain energy $W_{\varepsilon}$ and the rate of the 
stored energy $\rho \psi$ :

$$
\begin{aligned}
\dot{\Phi} & =\dot{W}_{\varepsilon}-\rho \dot{\psi} \geq 0 \\
& =\boldsymbol{\sigma}: \dot{\boldsymbol{\varepsilon}}-\rho\left(\frac{\partial \psi}{\partial \boldsymbol{\varepsilon}}: \dot{\boldsymbol{\varepsilon}}+\sum_{i=1}^{N} \frac{\partial \psi}{\partial \boldsymbol{\varepsilon}_{\boldsymbol{v}_{i}}}: \dot{\boldsymbol{\varepsilon}}_{\boldsymbol{v}_{i}}+\frac{\partial \psi}{\partial \boldsymbol{\varepsilon}_{\boldsymbol{p}}}: \dot{\boldsymbol{\varepsilon}}_{\boldsymbol{p}}+\frac{\partial \psi}{\partial r} \dot{r}+\frac{\partial \psi}{\partial D} \dot{D}\right) \geq 0 \\
& =\sum_{i=1}^{N} \boldsymbol{\sigma}_{\boldsymbol{v}_{i}}: \dot{\boldsymbol{\varepsilon}}_{\boldsymbol{v}_{i}}+\boldsymbol{\sigma}: \dot{\boldsymbol{\varepsilon}}_{\boldsymbol{p}}-R \dot{r}+Y \dot{D} \geq 0 .
\end{aligned}
$$

In order to satisfy the above inequality, the evolution laws expressing the rate of the internal state variables must be derived from a convex dual dissipation potential or a convex indicative function of the associated variables, in which the state variables themselves may act as parameters.

\subsubsection{Evolution law for viscoelasticity}

The evolution of each viscoelastic strain $\boldsymbol{\varepsilon}_{\boldsymbol{v}_{i}}$ is governed by a dual dissipation potential written as the sum of the sub-potentials of the linear dash-pot of each Kelvin-Voigt branch:

$$
\varphi^{*}\left(\boldsymbol{\sigma}_{\boldsymbol{v}_{i}} ; D\right)=\sum_{i=1}^{N} \varphi_{v_{i}}^{*}\left(\boldsymbol{\sigma}_{\boldsymbol{v}_{i}} ; D\right),
$$

with

$$
\varphi_{v_{i}}^{*}\left(\boldsymbol{\sigma}_{\boldsymbol{v}_{i}} ; D\right)=\frac{1}{2} \boldsymbol{\sigma}_{\boldsymbol{v}_{i}}: \frac{\mathbb{V}_{\boldsymbol{v}_{i}}^{-1}}{(1-D)}: \boldsymbol{\sigma}_{\boldsymbol{v}_{i}} .
$$

Then, the evolution of each viscoelastic strain $\boldsymbol{\varepsilon}_{\boldsymbol{v}_{i}}$ is given by differentiation of the pseudo-potential $\varphi^{*}$ with respect to its associated variable $\boldsymbol{\sigma}_{\boldsymbol{v}_{i}}$ :

$$
\dot{\boldsymbol{\varepsilon}}_{\boldsymbol{v}_{i}}=\frac{\partial \varphi^{*}}{\partial \boldsymbol{\sigma}_{\boldsymbol{v}_{i}}}=\frac{\mathbb{V}_{\boldsymbol{v}_{i}}^{-1}}{(1-D)}: \boldsymbol{\sigma}_{\boldsymbol{v}_{i}}
$$


The introduction of (8) into (17) finally allows to express the effective stress as a function of $\boldsymbol{\varepsilon}_{\boldsymbol{v}_{i}}$ and its rate:

$$
\frac{\boldsymbol{\sigma}}{1-D}=\mathbb{C}_{\boldsymbol{v}_{i}}: \boldsymbol{\varepsilon}_{\boldsymbol{v}_{i}}+\mathbb{V}_{\boldsymbol{v}_{i}}: \dot{\boldsymbol{\varepsilon}}_{\boldsymbol{v}_{i}}
$$

where $\mathbb{V}_{\boldsymbol{v}_{i}}$ denotes the viscosity tensor of the linear dash-pot of the $i^{\text {th }}$ KelvinVoigt branch. As for the stiffness tensors, each viscosity tensor is classically formulated for bulk isotropic materials and is defined by a viscosity $\eta_{v_{i}}$ and the Poisson ratio $\nu$. The latter is assumed to be the same in each stiffness and viscosity tensor. It is noted that the viscosity is introduced only in the dissipation potential and not in the Helmholtz free energy. This is always the case when viscoelasticity is described in differential form. In integral formulations, the viscocity is integrated directly in the Helmholtz free energy (Krairi and Doghri, 2014).

Within such a differential relationship (18), a characteristic time can be clearly identified for each Kelvin-Voigt branch by considering the creep test. Indeed, if a sudden constant effective stress $\widetilde{\boldsymbol{\sigma}}_{\mathbf{0}}$ is applied on a single KelvinVoigt branch at a time $t_{0}$ (Figure 2a), then it can be shown (Ottosen and Ristinmaa, 2005) that the strain response is approaching exponentially in time the strain of the pure elastic material $\boldsymbol{\varepsilon}_{\boldsymbol{v}_{i}}^{\infty}$ (Figure $2 \mathrm{~b}$ ):

$$
\boldsymbol{\varepsilon}_{\boldsymbol{v}_{i}}(t)=\boldsymbol{\varepsilon}_{\boldsymbol{v}_{i}}^{\infty}\left(1-e^{-\frac{t-t_{0}}{\tau_{v_{i}}}}\right) \quad \text { with } \quad \varepsilon_{\boldsymbol{v}_{i}}^{\infty}=\mathbb{C}_{\boldsymbol{v}_{i}}^{-1}: \tilde{\boldsymbol{\sigma}}_{\mathbf{0}}
$$

where $\tau_{v_{i}}$ is the characteristic time given by:

$$
\tau_{v_{i}} \mathbb{I}=\mathbb{C}_{\boldsymbol{v}_{i}}^{-1}: \mathbb{V}_{\boldsymbol{v}_{i}} \quad \text { or } \quad \tau_{v_{i}}=\frac{\eta_{v_{i}}}{E_{v_{i}}}
$$


From a mathematical point of view, $\tau_{v_{i}}$ corresponds to the time needed to reach $63 \%\left(1-e^{-1}=0.63\right)$ of the pure elastic strain $\boldsymbol{\varepsilon}_{\boldsymbol{v}_{i}}^{\infty}$ (Figure $\left.2 \mathrm{~b}\right)$. Basically, the characteristic time provides an information about which time range a Kelvin-Voigt branch is expected to move.

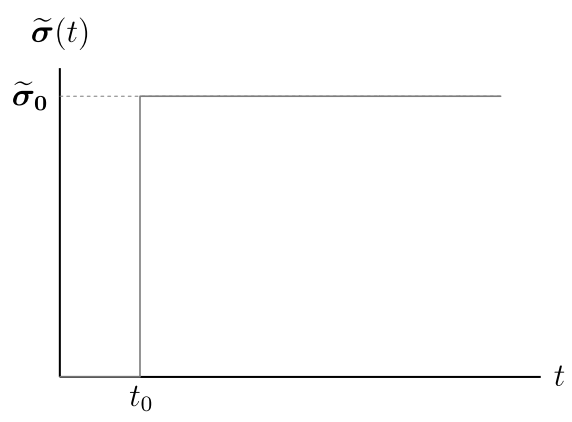

(a) Application of a sudden constant effective stress

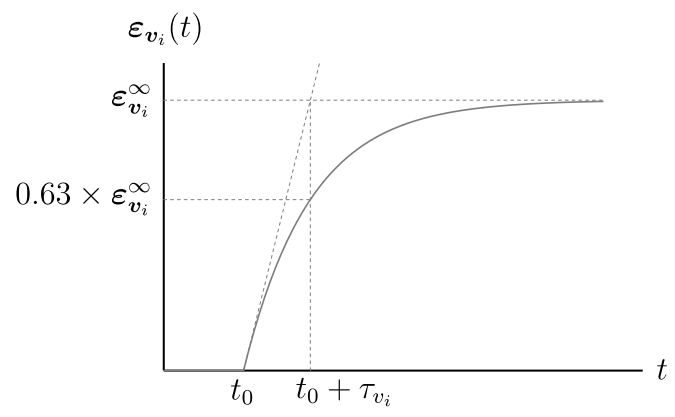

(b) Strain response

Figure 2: Illustration of the behaviour of a single Kelvin-Voigt branch through the creep test.

Considering any type of loading conditions as being a continuous succession of creep tests, the Boltzmann superposition principle (Ottosen and Ristinmaa, 2005) allows to express the evolution of each viscoelastic strain $\varepsilon_{v_{i}}$ from an integral representation that involves the whole history of the effective stress:

$$
\boldsymbol{\varepsilon}_{\boldsymbol{v}_{i}}(t)=\int_{-\infty}^{t} \frac{\mathbb{C}_{\boldsymbol{v}_{i}}^{-1}}{1-D(\xi)}\left(1-e^{-\frac{t-\xi}{\tau v_{i}}}\right): \frac{\partial \boldsymbol{\sigma}(\xi)}{\partial \xi} d \xi .
$$

Thus, the relations governing each viscoelastic strain may be expressed either in differential (18) or integral (21) forms. Both representations have advantages and drawbacks as previously discussed in the introductory section (Section 1). The integral formulation in (21) along with the relationships 
(19) and (20) are only valid if the stiffness and viscosity tensors $\mathbb{C}_{\boldsymbol{v}_{i}}$ and $\mathbb{V}_{\boldsymbol{v}_{i}}$, respectively, are defined as isotropic with the same Poisson ratio. However, it is worth noticing that the differential form (18) provides more freedom regarding the expressions of $\mathbb{C}_{\boldsymbol{v}_{i}}$ and $\mathbb{V}_{\boldsymbol{v}_{i}}$. With this formalism, anisotropy can be integrated in a straightforward manner. In this work, it is recalled that the differential representation (18) has been adopted.

\subsubsection{Evolution laws for coupled viscoplasticity and damage}

As previously mentioned, viscoplasticity and damage are considered as coupled phenomena (Lemaitre, 1985; Lemaitre and Desmorat, 2005; Krairi and Doghri, 2014). Such a connection requires a non associative mechanism. Consequently, the evolution of $r, \varepsilon_{p}$ and $D$ cannot be obtained from a dual dissipation potential. However, the evolution laws are expressed by the normality of a an indicative function given by:

$$
F(\boldsymbol{\sigma}, R, Y ; D)=f(\boldsymbol{\sigma}, R ; D)+f_{D}(Y ; D) .
$$

In the above equation, $f(\boldsymbol{\sigma}, R ; D)$ is the yield criterion function that is expressed in the effective stress space in order to account the coupling between damage and viscoplasticity:

$$
f(\boldsymbol{\sigma}, R ; D)=\frac{e q(\boldsymbol{\sigma})}{1-D}-R-R_{0},
$$

where $R_{0}$ is the yield threshold. The part related to damage denoted by $f_{D}(Y ; D)$ is given by:

$$
f_{D}(Y ; D)=\frac{S}{(\beta+1)(1-D)}\left(\frac{Y}{S}\right)^{\beta+1},
$$


where $S$ and $\beta$ are material parameters. Then, as previously mentioned, the evolution of $r, \varepsilon_{p}$ and $D$ are given by the normality of the indicative function $F(\boldsymbol{\sigma}, R, Y ; D)$ that introduces a viscoplastic multiplier $\lambda$ :

$$
\begin{gathered}
\dot{r}=-\frac{\partial F}{\partial R} \dot{\lambda}=\dot{\lambda} \\
\dot{\boldsymbol{\varepsilon}}_{\boldsymbol{p}}=\frac{\partial F}{\partial \boldsymbol{\sigma}} \dot{\lambda}=\frac{3}{2} \frac{\boldsymbol{d e} \boldsymbol{v}(\boldsymbol{\sigma})}{e q(\boldsymbol{\sigma})} \frac{\dot{\lambda}}{1-D}, \\
\dot{D}=\frac{\partial F}{\partial Y} \dot{\lambda}=\left(\frac{Y}{S}\right)^{\beta} \frac{\dot{\lambda}}{1-D} .
\end{gathered}
$$

The introduction of (25) into (26) and (27) allows to express the evolution of $\varepsilon_{p}$ and $D$ under the form of flow equations:

$$
\dot{\varepsilon}_{p}=\frac{\boldsymbol{\Lambda}(\boldsymbol{\sigma})}{1-D} \dot{r}, \quad \dot{D}=\frac{\Omega(Y)}{1-D} \dot{r}
$$

where $\boldsymbol{\Lambda}(\boldsymbol{\sigma})$ and $\Omega(Y)$ are given by:

$$
\boldsymbol{\Lambda}(\boldsymbol{\sigma})=\frac{3}{2} \frac{\boldsymbol{d e} \boldsymbol{v}(\boldsymbol{\sigma})}{e q(\boldsymbol{\sigma})}, \quad \Omega(Y)=\left(\frac{Y}{S}\right)^{\beta} .
$$

Note that, for semi-crystalline polymers the damage growth appears to be relatively fast at the beginning of a loading path and gradually decreases as the loading continues (Detrez et al., 2011). In order to account for this experimentally observed tendency, the parameter $\beta$ in (24) and (29) must be negative. When $\beta$ is positive the inverse trend is obtained (slow initiation and faster subsequent growth), which is frequently observed in metals (Krairi and Doghri, 2014).

The evolution of the multiplier $\dot{\lambda}=\dot{r}$ is activated $(\dot{r}>0)$ only if the criterion function $f$ becomes positive. Next, in order to account for the 
visoplasticity, rate dependency is introduced by considering that the positive part of $f$ is equal to the effective equivalent stress acting on the non-linear dash-pot of the viscoplastic branch (Figure 1c). This stress is connected to the rate of $r$ through the function $Q(\dot{r})$. This finally gives a relationship between the effective equivalent stress, the variable $r$ and its rate:

$$
\langle\underbrace{\frac{e q(\boldsymbol{\sigma})}{1-D}-R(r)-R_{0}}_{f}\rangle_{+}=Q(\dot{r}) .
$$

The function $Q(\dot{r})$ must be increasing, positive and null at $\dot{r}=0$. In this model, this function is chosen under the form of a power law:

$$
Q(\dot{r})=H \dot{r}^{m}
$$

where $H$ and $m$ are material parameters. It is worth noticing that the value of $r$ can only increase $(\dot{r}>0$ if $f>0)$ or remain constant $(\dot{r}=0$ if $f<0)$. Consequently, the part of the stored energy related to the viscoplastic branch $\psi_{p}$ written in (6), appears to be irrecoverable, as it cannot return back to zero.

\section{Numerical implementation: Backward Euler time implicit algo- rithm}

Based on a backward Euler time implicit numerical scheme, the value of a given quantity $x$ is updated from the previous time step $n$ to the current $n+1$ per $x^{(n+1)}=x^{(n)}+\Delta x^{(n+1)}$. Such an implicit relation is usually solved iteratively, and the current value is updated for each iteration $k$ by: $x^{(n+1)(k+1)}=x^{(n+1)(k)}+\delta x^{(n+1)(k)}$ until $x^{(n+1)}$ has converged. Obviously 
$\Delta x^{(n+1)(k+1)}=\Delta x^{(n+1)(k)}+\delta x^{(n+1)(k)}$.

When designing a constitutive law for a finite element software like ABAQUS (UMAT subroutine), the main idea is that, at the time increment $n$, the analysis is completed and the finite element code provides i) all the variables at the time increment $n$ and ii) the increment of total strain $\Delta \varepsilon^{(n+1)}$. The aim of a UMAT subroutine is to compute a) the stress and the state variables at time step $n+1$, and b) the tangent operator $\mathbb{C}_{\boldsymbol{t}}$ for the next finite element calculation. Since for each loading step the total strain increment $\Delta \varepsilon^{(n+1)}$ is provided by the global solver and is thus known, it is the role of the UMAT subroutine to find the current stress. During iterative correction, the total strain at the current time step is considered constant such that $\delta \varepsilon^{(n+1)(k)}=0$.

Such type of time implicit implementation requires a "return mapping algorithm", from which two main forms are commonly used, namely, the "closest point projection" and the "convex cutting plane" (Ortiz and Simo, 1986; Simo and Hughes, 1998). The comparison between these two methods (Qidwai and Lagoudas, 2000) pointed out that, the "convex cutting plane", in addition to its simplicity, involves less tensorial operations and computational cost than the "closest point projection" while both provide satisfactory results. For this reason, the proposed implementation is based on the "convex cutting plane" method.

To consider such type of numerical scheme it is convenient to write the equations governing the evolutions of the state variables under the form of 
residual functions that must satisfy a nullity condition. In the case of the proposed model, the evolution law of each viscoelastic strain $\boldsymbol{\varepsilon}_{\boldsymbol{v}_{\boldsymbol{i}}}$ (18) is written under the form of a tensorial function $\boldsymbol{\phi}_{\boldsymbol{v}_{i}}$ that must always remain null as the viscoelastic mechanisms are always active:

$$
\phi_{\boldsymbol{v}_{i}}=\dot{\boldsymbol{\varepsilon}}_{\boldsymbol{v}_{i}}-\mathbb{V}_{\boldsymbol{v}_{i}}^{-1}:\left(\frac{\boldsymbol{\sigma}}{1-D}-\mathbb{C}_{\boldsymbol{v}_{i}}: \boldsymbol{\varepsilon}_{\boldsymbol{v}_{i}}\right)=\mathbf{0} .
$$

In the same principle, the evolution law governing viscoplasticity and damage through the variable $r(30)$ is written under the form of a scalar function $\phi_{r}$ that must remains null as long as $f$ is positive as this mechanisms is subjected to a Kuhn Tucker condition: it is only activated if $f$ is positive:

$$
\phi_{r}=\dot{r}-Q^{-1}(\underbrace{\frac{e q(\boldsymbol{\sigma})}{1-D}-R(r)-R_{0}}_{f>0})=0 .
$$

$Q^{-1}(f)$ is the inverse function of $Q(\dot{r})$ that is defined in (31). It is precised that, within the "convex cutting plane" method, the integration of the flow equations (28) as residual functions is not required.

The procedure of the algorithms is described as follows:

- In the first step, usually called the "elastic prediction", all the internal state variables are assumed not to evolve and only generation of elastic strain is considered. Thus, during this step, only the single spring is acting and the strain increment provided by the solver of the equilibrium equations is considered to be elastic $\left(\Delta \varepsilon=\Delta \varepsilon_{e}\right)$.

- In the second step called the "viscoelastic correction/prediction", the total strain is fixed, and the error in the stress is corrected by developing 
only the viscoelastic strains $\boldsymbol{\varepsilon}_{\boldsymbol{v}_{i}}$. Thus, during this step, only the single spring and the Kelvin-Voigt branches are acting. The total strain $\varepsilon$ does not evolve, while the viscoelastic strains $\boldsymbol{\varepsilon}_{\boldsymbol{v}_{i}}$ are generated by considering the nullity of all the residuals $\boldsymbol{\phi}_{\boldsymbol{v}_{i}}$ at the same time.

- The third step called the "full correction" is only required if the criterion function $f$ is positive at the end of the second step. In the same way, the total strain $\varepsilon$ is fixed, and the error in the stress is corrected by developing all the internal state variables $\left(\boldsymbol{\varepsilon}_{\boldsymbol{v}_{i}}, \boldsymbol{\varepsilon}_{\boldsymbol{p}}, r\right.$ and $\left.D\right)$. Thus, during this step, all the branches are active. The total strain $\varepsilon$ does not evolve, while all the internal state variables $\left(\boldsymbol{\varepsilon}_{\boldsymbol{v}_{i}}, \boldsymbol{\varepsilon}_{\boldsymbol{p}}, r\right.$ and $\left.D\right)$ are generated by considering the nullity of all the residuals $\boldsymbol{\phi}_{\boldsymbol{v}_{i}}$ and $\phi_{r}$ at the same time.

The second and third steps require the numerical integration of the evolution equations through a "Newton-Raphson scheme". They also require the identification of the tangent operator that is necessary for the finite element framework.

\subsection{Elastic prediction}

For the "elastic prediction", the stress is predicted by assuming that the internal state variables do not evolve. Thus only generation of elastic strain is considered. The total strain at the end of the increment is given by $\varepsilon^{(n+1)}=\varepsilon^{(n)}+\Delta \varepsilon^{(n+1)}$ while the internal state variables increments are considered to be null, such as: $\Delta \boldsymbol{\varepsilon}_{\boldsymbol{v}_{i}}{ }^{(n+1)(k=0)}=\mathbf{0}, \Delta r^{(n+1)(k=0)}=0$, $\Delta \boldsymbol{\varepsilon}_{\boldsymbol{p}}{ }^{(n+1)(k=0)}=\mathbf{0}$ and $\Delta D^{(n+1)(k=0)}=0$. At the end of this step, the nullity 
of the residuals $\phi_{\boldsymbol{v}_{i}}$ cannot be respected, then the stress must be corrected in order to satisfy this condition. It is the role of the next step called the "viscoelastic correction/prediction".

\subsection{Viscoelastic correction/prediction}

In the "viscoelastic correction/prediction" step, only the viscoelastic strains $\varepsilon_{v_{i}}$ evolve while the other internal state variables are still assumed to be unchanged as it is not known yet whether the material is actively yielding or not, consequently: $\delta r^{(n+1)(k)}=0, \delta \varepsilon_{p}{ }^{(n+1)(k)}=\mathbf{0}$ and $\delta D^{(n+1)(k)}=0$. The "visoelastic correction/prediction" consists in forcing the functions $\phi_{v_{i}}$ to be null by developing only the viscoelastic strains $\boldsymbol{\varepsilon}_{\boldsymbol{v}_{i}}$ while the total strain does not evolve such that $\delta \boldsymbol{\varepsilon}^{(n+1)(k)}=0$. To do so, the nullity condition of each function $\phi_{\boldsymbol{v}_{\boldsymbol{i}}}$ is expressed by:

$$
\boldsymbol{\phi}_{\boldsymbol{v}_{i}}^{(n+1)(k+1)}=\boldsymbol{\phi}_{\boldsymbol{v}_{i}}^{(n+1)(k)}+\delta \boldsymbol{\phi}_{\boldsymbol{v}_{i}}^{(n+1)(k)}=\mathbf{0},
$$

where $\delta \boldsymbol{\phi}_{\boldsymbol{v}_{i}}^{(n+1)(k)}$ is computed by linearisation. After proper calculation (Appendix B), it can be expressed under the following form (all the quantities are taken at the increment $\left.{ }^{(n+1)(k)}\right)$ :

$$
\delta \phi_{v_{i}}=\mathbb{A}_{\boldsymbol{v}_{i} \boldsymbol{\sigma}}: \delta \boldsymbol{\sigma}+\mathbb{A}_{\boldsymbol{v}_{i} \boldsymbol{v}_{i}}: \delta \boldsymbol{\varepsilon}_{\boldsymbol{v}_{i}}
$$

In the same manner (Appendix B), $\delta \boldsymbol{\sigma}$ is written as:

$$
\delta \boldsymbol{\sigma}=\sum_{j=1}^{N} \mathbb{B}_{\boldsymbol{\sigma} \boldsymbol{v}_{j}}: \delta \boldsymbol{\varepsilon}_{\boldsymbol{v}_{j}}
$$

By introducing (36) into (35) while considering (34), the unknown values of $\delta \varepsilon_{\boldsymbol{v}_{i}}$ are computed from the known residuals $\phi_{\boldsymbol{v}_{\boldsymbol{i}}}$ through the construction of 
the following "viscoelastic corrector system" whose the expression is detailed in Appendix C):

$$
\left\{\begin{array}{c}
\delta \boldsymbol{\varepsilon}_{\boldsymbol{v}_{1}} \\
\delta \boldsymbol{\varepsilon}_{\boldsymbol{v}_{2}} \\
\delta \boldsymbol{\varepsilon}_{\boldsymbol{v}_{3}} \\
\vdots \\
\delta \boldsymbol{\varepsilon}_{\boldsymbol{v}_{N}}
\end{array}\right\}=\left(\begin{array}{ccccc}
\mathbb{L}_{\boldsymbol{v}_{1} \boldsymbol{v}_{1}}^{*} & \mathbb{L}_{\boldsymbol{v}_{1} \boldsymbol{v}_{2}}^{*} & \mathbb{L}_{\boldsymbol{v}_{1} \boldsymbol{v}_{3}}^{*} & \ldots & \mathbb{L}_{\boldsymbol{v}_{1} \boldsymbol{v}_{N}}^{*} \\
\mathbb{L}_{\boldsymbol{v}_{2} \boldsymbol{v}_{1}}^{*} & \mathbb{L}_{\boldsymbol{v}_{2} \boldsymbol{v}_{2}}^{*} & \mathbb{L}_{\boldsymbol{v}_{2} \boldsymbol{v}_{3}}^{*} & \ldots & \mathbb{L}_{\boldsymbol{v}_{2} \boldsymbol{v}_{N}}^{*} \\
\mathbb{L}_{\boldsymbol{v}_{3} \boldsymbol{v}_{1}}^{*} & \mathbb{L}_{\boldsymbol{v}_{3} \boldsymbol{v}_{2}}^{*} & \mathbb{L}_{\boldsymbol{v}_{3} \boldsymbol{v}_{3}}^{*} & \ldots & \mathbb{L}_{\boldsymbol{v}_{3} \boldsymbol{v}_{N}}^{*} \\
\vdots & \vdots & \vdots & \ddots & \vdots \\
\mathbb{L}_{\boldsymbol{v}_{N} \boldsymbol{v}_{1}}^{*} & \mathbb{L}_{\boldsymbol{v}_{N} \boldsymbol{v}_{2}}^{*} & \mathbb{L}_{\boldsymbol{v}_{N} \boldsymbol{v}_{3}}^{*} & \ldots & \mathbb{L}_{\boldsymbol{v}_{N} \boldsymbol{v}_{N}}^{*}
\end{array}\right) \times\left\{\begin{array}{c}
-\boldsymbol{\phi}_{\boldsymbol{v}_{1}} \\
-\boldsymbol{\phi}_{\boldsymbol{v}_{2}} \\
-\boldsymbol{\phi}_{\boldsymbol{v}_{3}} \\
\vdots \\
-\boldsymbol{\phi}_{\boldsymbol{v}_{N}}
\end{array}\right\} .
$$

Thus, using the above relationship, the viscoelastic strains $\boldsymbol{\varepsilon}_{\boldsymbol{v}_{i}}$ are updated at the increment ${ }^{(n+1)(k+1)}$. Finally, the residuals $\boldsymbol{\phi}_{\boldsymbol{v}_{i}}$ are checked in order to know whether the correction is sufficient or not:

- If $\left|\boldsymbol{\phi}_{\boldsymbol{v}_{i}}^{(n+1)(k+1)}\right| \leq 0+\delta$, then the stress can be considered as corrected and the algorithm proceeds to the next step.

- If $\left.\mid \boldsymbol{\phi}_{\boldsymbol{v}_{i}}^{(n+1)(k+1)}\right) \mid>0+\delta$, then the stress remains inaccurate. One or several correction loops ( $k$ loop) are required to reach the convergence (until $\left|\boldsymbol{\phi}_{\boldsymbol{v}_{i}}^{(n+1)(k+1)}\right| \leq 0+\delta$ ) before going to the next step.

Normally, as in the present model the viscoelastic mechanisms are linear, it can be shown that only one correction is sufficient to reach the convergence. However, this scheme can also be applied to non-linear viscoelasticity where several corrections would be likely required.

Once the convergence has been reached $\left(\left|\boldsymbol{\phi}_{\boldsymbol{v}_{i}}^{(n+1)(k+1)}\right| \leq 0+\delta\right)$, the criterion function $f$ is checked in order to identify whether the material is actively yielding or not: 
- If $f^{(n+1)(k+1)} \leq 0$, then the material is not actively yielding and the stress is not corrected. Consequently, the "full correction" step is not required and the algorithm can proceed directly to the next total strain increment.

- If $f^{(n+1)(k+1)}>0$, then the material is actively yielding and the residual $\phi_{r}$ is computed. Moreover, the stress needs to be corrected again with the "full correction" where all the internal state variables will be evaluated in order to keep the residuals $\boldsymbol{\phi}_{\boldsymbol{v}_{i}}$ and $\phi_{r}$ null at the same time.

\subsection{Full correction}

The "full correction" step consists in forcing both residuals $\boldsymbol{\phi}_{\boldsymbol{v}_{i}}$ and $\phi_{r}$ to be null by developing all the internal state variables, while the total strain still does not evolve such that $\delta \varepsilon^{(n+1)(k)}=0$. As previously mentioned, the proposed implementation is based on the "convex cutting plane" method (Ortiz and Simo, 1986; Simo and Hughes, 1998), where the flow equations (28) are explicitly integrated within the correction procedure, while the time inte-

gration remains implicit. According to this scheme, the quantities $\delta \varepsilon_{p}^{(n+1)(k)}$ and $\delta D^{(n+1)(k)}$ are directly linked to $\delta r^{(n+1)(k)}$ by:

$$
\delta \varepsilon_{\boldsymbol{p}}^{(n+1)(k)}=\frac{\boldsymbol{\Lambda}\left(\boldsymbol{\sigma}^{(n+1)(k)}\right)}{1-D^{(n+1)(k)}} \delta r^{(n+1)(k)}, \quad \delta D^{(n+1)(k)}=\frac{\Omega\left(Y^{(n+1)(k)}\right)}{1-D^{(n+1)(k)}} \delta r^{(n+1)(k)} .
$$

The nullity conditions of $\boldsymbol{\phi}_{\boldsymbol{v}_{i}}$ and $\phi_{r}$ are expressed by:

$$
\left\{\begin{array}{l}
\phi_{\boldsymbol{v}_{i}}^{(n+1)(k+1)}=\phi_{\boldsymbol{v}_{i}}^{(n+1)(k)}+\delta \phi_{\boldsymbol{v}_{i}}^{(n+1)(k)}=0 \\
\phi_{r}^{(n+1)(k+1)}=\phi_{r}^{(n+1)(k)}+\delta \phi_{r}^{(n+1)(k)}=0
\end{array},\right.
$$


where $\delta \phi_{\boldsymbol{v}_{i}}^{(n+1)(k)}$ and $\delta \phi_{r}^{(n+1)(k)}$ are computed by linearisation. After proper calculation (Appendix B), they can be expressed under the following form (all the quantities are taken at the increment $\left.{ }^{(n+1)(k)}\right)$ :

$$
\left\{\begin{array}{l}
\delta \phi_{\boldsymbol{v}_{i}}=\mathbb{A}_{\boldsymbol{v}_{i} \boldsymbol{\sigma}}: \delta \boldsymbol{\sigma}+\mathbb{A}_{\boldsymbol{v}_{i} \boldsymbol{v}_{i}}: \delta \boldsymbol{\varepsilon}_{\boldsymbol{v}_{i}}+\boldsymbol{A}_{\boldsymbol{v}_{i} \boldsymbol{r}} \delta r \\
\delta \phi_{r}=\boldsymbol{A}_{\boldsymbol{r} \boldsymbol{\sigma}}: \delta \boldsymbol{\sigma}+A_{r r} \delta r
\end{array}\right.
$$

In the same manner (Appendix B), $\delta \boldsymbol{\sigma}$ is written as:

$$
\delta \boldsymbol{\sigma}=\sum_{j=1}^{N} \mathbb{B}_{\boldsymbol{\sigma} \boldsymbol{v}_{j}}: \delta \boldsymbol{\varepsilon}_{\boldsymbol{v}_{j}}+\boldsymbol{B}_{\boldsymbol{\sigma} \boldsymbol{r}} \delta r .
$$

By introducing (41) into (40) while considering (39), the unknown values of $\delta \boldsymbol{\varepsilon}_{\boldsymbol{v}_{i}}$ and $\delta r$ are computed from the known residuals $\boldsymbol{\phi}_{\boldsymbol{v}_{\boldsymbol{i}}}$ and $\phi_{r}$ through the construction of the following "full corrector system" whose the expression is detailed in Appendix C):

$$
\left\{\begin{array}{c}
\delta \boldsymbol{\varepsilon}_{\boldsymbol{v}_{1}} \\
\delta \boldsymbol{\varepsilon}_{\boldsymbol{v}_{2}} \\
\delta \boldsymbol{\varepsilon}_{\boldsymbol{v}_{3}} \\
\vdots \\
\delta \boldsymbol{\varepsilon}_{\boldsymbol{v}_{N}} \\
\delta r
\end{array}\right\}=\left(\begin{array}{cccccc}
\mathbb{L}_{\boldsymbol{v}_{1} \boldsymbol{v}_{1}} & \mathbb{L}_{\boldsymbol{v}_{1} \boldsymbol{v}_{2}} & \mathbb{L}_{\boldsymbol{v}_{1} \boldsymbol{v}_{3}} & \ldots & \mathbb{L}_{\boldsymbol{v}_{1} \boldsymbol{v}_{N}} & \boldsymbol{L}_{\boldsymbol{v}_{1} \boldsymbol{r}} \\
\mathbb{L}_{\boldsymbol{v}_{2} \boldsymbol{v}_{1}} & \mathbb{L}_{\boldsymbol{v}_{2} \boldsymbol{v}_{2}} & \mathbb{L}_{\boldsymbol{v}_{2} \boldsymbol{v}_{3}} & \ldots & \mathbb{L}_{\boldsymbol{v}_{2} \boldsymbol{v}_{N}} & \boldsymbol{L}_{\boldsymbol{v}_{2} \boldsymbol{r}} \\
\mathbb{L}_{\boldsymbol{v}_{3} \boldsymbol{v}_{1}} & \mathbb{L}_{\boldsymbol{v}_{3} \boldsymbol{v}_{2}} & \mathbb{L}_{\boldsymbol{v}_{3} \boldsymbol{v}_{3}} & \ldots & \mathbb{L}_{\boldsymbol{v}_{3} \boldsymbol{v}_{N}} & \boldsymbol{L}_{\boldsymbol{v}_{3} \boldsymbol{r}} \\
\vdots & \vdots & \vdots & \ddots & \vdots & \vdots \\
\mathbb{L}_{\boldsymbol{v}_{N} \boldsymbol{v}_{1}} & \mathbb{L}_{\boldsymbol{v}_{N} \boldsymbol{v}_{2}} & \mathbb{L}_{\boldsymbol{v}_{N} \boldsymbol{v}_{3}} & \ldots & \mathbb{L}_{\boldsymbol{v}_{N} \boldsymbol{v}_{N}} & \boldsymbol{L}_{\boldsymbol{v}_{N} \boldsymbol{r}} \\
\boldsymbol{L}_{\boldsymbol{r} \boldsymbol{v}_{1}} & \boldsymbol{L}_{\boldsymbol{r} \boldsymbol{v}_{2}} & \boldsymbol{L}_{\boldsymbol{r} \boldsymbol{v}_{3}} & \ldots & \boldsymbol{L}_{\boldsymbol{r} \boldsymbol{v}_{N}} & L_{r r}
\end{array}\right) \times\left\{\begin{array}{c}
-\boldsymbol{\phi}_{\boldsymbol{v}_{1}} \\
-\boldsymbol{\phi}_{\boldsymbol{v}_{2}} \\
-\boldsymbol{\phi}_{\boldsymbol{v}_{3}} \\
\vdots \\
-\boldsymbol{\phi}_{\boldsymbol{v}_{N}} \\
-\phi_{r}
\end{array}\right\} .
$$

Thus, using the above relationship along with (38), the variables $\boldsymbol{\varepsilon}_{\boldsymbol{v}_{i}}, r, \boldsymbol{\varepsilon}_{\boldsymbol{p}}$ and $D$ are updated at the increment ${ }^{(n+1)(k+1)}$. Finally, the residuals $\boldsymbol{\phi}_{\boldsymbol{v}_{i}}$ and $\phi_{r}$ are checked in order to identify whether the correction is sufficient or not:

- If $\left|\phi_{\boldsymbol{v}_{i}}^{(n+1)(k+1)}\right|$ and $\left|\phi_{r}^{(n+1)(k+1)}\right| \leq 0+\delta$, then the stress can be considered as corrected and the algorithm can proceed to the next total strain increment. 
- If $\left|\boldsymbol{\phi}_{\boldsymbol{v}_{i}}^{(n+1)(k+1)}\right|$ and $\left|\phi_{r}^{(n+1)(k+1)}\right|>0+\delta$, then the stress remains inaccurate. One or several correction loops ( $k$ loop) are required to reach the convergence (until $\left|\boldsymbol{\phi}_{\boldsymbol{v}_{i}}^{(n+1)(k+1)}\right| \leq 0+\delta$ and $\left|\phi_{r}^{(n+1)(k+1)}\right| \leq 0+\delta$ ) before passing to the next total strain increment.

\subsection{Tangent operator}

In addition to computing the updated stress, the global finite element solver also requires the tangent operator $\mathbb{C}_{t}$ which defines the current rate of change of stress with a change in total strain. It is explained in the literature (Simo and Hughes, 1998) that the "convex cutting plane" form of the "return mapping algorithm" utilizes the continuum tangent operator. Its scope is to identify the relation between $d \boldsymbol{\sigma}$ and $d \boldsymbol{\varepsilon}$ using the continuum description of the algorithm. To do so, the stress-strain relation is rewritten in differential form and the evolution equations are substituted. With the proposed model, two configurations of tangent operator are to be taken into account:

- When only viscoelastic strain is generated. The "return mapping algorithm" stopped at the "viscoelastic correction/prediction" step. In this case, the "viscoelastic tangent operator" $\mathbb{C}_{t}^{*}$ is used.

- When both viscoelasticity, viscoplasticity and damage are activated. The "return mapping algorithm" went through the "full correction" step. In this case, the "full tangent operator" $\mathbb{C}_{t}$ is used. 


\subsubsection{Viscoelastic tangent operator}

When only viscoelastic strain is generated, the linearised stress-strain relationship can be written as (Appendix B):

$$
d \boldsymbol{\sigma}=\mathbb{B}_{\boldsymbol{\sigma} \varepsilon}: d \boldsymbol{\varepsilon}+\sum_{j=1}^{N} \mathbb{B}_{\boldsymbol{\sigma} \boldsymbol{v}_{j}}: d \boldsymbol{\varepsilon}_{\boldsymbol{v}_{j}} .
$$

The constraint equations $d \boldsymbol{\phi}_{\boldsymbol{v}_{i}}=0$ must be satisfied for all acceptable solutions. Expressed in its linearised form (Appendix B), this gives:

$$
d \phi_{\boldsymbol{v}_{i}}=\mathbb{A}_{\boldsymbol{v}_{i} \boldsymbol{\sigma}}: d \boldsymbol{\sigma}+\mathbb{A}_{\boldsymbol{v}_{i} \boldsymbol{v}_{i}}: d \boldsymbol{\varepsilon}_{\boldsymbol{v}_{i}}=0 .
$$

By introducing (43) into (44), a linear relationship can be identified between each $d \boldsymbol{\varepsilon}_{\boldsymbol{v}_{j}}$ and $d \boldsymbol{\varepsilon}$, such that:

$$
d \varepsilon_{\boldsymbol{v}_{j}}=\mathbb{X}_{\boldsymbol{v}_{j} \varepsilon}^{*}: d \varepsilon
$$

where the terms $\mathbb{X}_{v_{j} \varepsilon}^{*}$ are the "tangent multipliers" whose expressions are

provided in Appendix D. Finally, the substitution of (45) into (43) leads to the identification of the "viscoelastic tangent operator" such that $d \boldsymbol{\sigma}=\mathbb{C}_{t}^{*}$ : $d \boldsymbol{\varepsilon}$. Its formulation is then given by:

$$
\mathbb{C}_{t}^{*}=\mathbb{B}_{\boldsymbol{\sigma} \varepsilon}+\sum_{j=1}^{N} \mathbb{B}_{\boldsymbol{\sigma} \boldsymbol{v}_{j}}: \mathbb{X}_{\boldsymbol{v}_{j} \varepsilon}^{*} .
$$

\subsubsection{Full tangent operator}

When both viscoelasticity, viscoplasticity and damage are activated, the linearised stress-strain relationship can be written as (Appendix B):

$$
d \boldsymbol{\sigma}=\mathbb{B}_{\boldsymbol{\sigma} \varepsilon}: d \boldsymbol{\varepsilon}+\sum_{j=1}^{N} \mathbb{B}_{\boldsymbol{\sigma} \boldsymbol{v}_{j}}: d \boldsymbol{\varepsilon}_{\boldsymbol{v}_{j}}+\boldsymbol{B}_{\boldsymbol{\sigma} \boldsymbol{r}} d r .
$$


The constraint equations $d \boldsymbol{\phi}_{\boldsymbol{v}_{i}}=0$ and $d \phi_{r}=0$ must be both satisfied for all acceptable solutions. Expressed in their linearised forms (Appendix B), this gives:

$$
\left\{\begin{array}{l}
d \boldsymbol{\phi}_{\boldsymbol{v}_{i}}=\mathbb{A}_{\boldsymbol{v}_{i} \boldsymbol{\sigma}}: d \boldsymbol{\sigma}+\mathbb{A}_{\boldsymbol{v}_{i} \boldsymbol{v}_{i}}: d \boldsymbol{\varepsilon}_{\boldsymbol{v}_{i}}+\boldsymbol{A}_{\boldsymbol{v}_{i} \boldsymbol{r}} d r=0 \\
d \phi_{r}=\boldsymbol{A}_{\boldsymbol{r} \boldsymbol{\sigma}}: d \boldsymbol{\sigma}+A_{r r} d r=0
\end{array} .\right.
$$

By introducing (47) into (48), a linear relationship can be identified between each $d \boldsymbol{\varepsilon}_{\boldsymbol{v}_{j}}$ and $d \boldsymbol{\varepsilon}$, and between $d r$ and $d \boldsymbol{\varepsilon}$, such that:

$$
\left\{\begin{array}{l}
d \boldsymbol{\varepsilon}_{\boldsymbol{v}_{j}}=\mathbb{X}_{\boldsymbol{v}_{j} \varepsilon}: d \boldsymbol{\varepsilon} \\
d r=\boldsymbol{X}_{\boldsymbol{r} \varepsilon}: d \varepsilon
\end{array}\right.
$$

where the terms $\mathbb{X}_{\boldsymbol{v}_{j} \varepsilon}$ and $\boldsymbol{X}_{\boldsymbol{r} \varepsilon}$ are the "tangent multipliers" whose expressions are provided in Appendix D. Finally, the substitution of (49) into (47) leads to the identification of the "full tangent operator" such that $d \boldsymbol{\sigma}=\mathbb{C}_{\boldsymbol{t}}: d \varepsilon$. Its formulation is then given by:

$$
\mathbb{C}_{\boldsymbol{t}}=\mathbb{B}_{\boldsymbol{\sigma} \varepsilon}+\sum_{j=1}^{N} \mathbb{B}_{\boldsymbol{\sigma} \boldsymbol{v}_{j}}: \mathbb{X}_{\boldsymbol{v}_{j} \varepsilon}+\boldsymbol{B}_{\boldsymbol{\sigma} \boldsymbol{r}} \otimes \boldsymbol{X}_{\boldsymbol{r} \varepsilon}
$$

\section{Experimental identification, strategy and validation}

\subsection{Experimental procedure and testing}

In this section, it is proposed to apply the previously formulated constitutive model to describe the behaviour of the polyamide $(6,6)$. This type of material is well known to be highly sensitive to the environmental conditions (Launay et al., 2013c; Arif et al., 2014a), especially the relative humidity $(R H)$ and the temperature $(T)$. In the present case, the following environmental conditions were considered: $R H=50 \%$ and $T=23^{\circ} \mathrm{C}$ (room temperature). For the experimental program, uni-axial tests were carried 
out on ISO527-2-1A tensile specimens (Figure 3). A particular attention has been paid concerning the $R H$ conditioning prior to performing the mechanical testing. The samples were placed in a oven with an air containing $50 \%$ of relative humidity at the temperature of $70^{\circ} \mathrm{C}$ until the equilibrium in water concentration was reached within the material. This was checked by regularly weighting the samples until their mass does not evolve any more. Next, the samples were placed in a sealed bag before testing. The tensile tests were performed on a ZWICK ROELL servo-hydraulic tensile machine at room temperature with a local strain measurement by means of an extensometer. It is also pointed out that all the tests were controlled by moving the crosshead with a nominal strain rate that may slightly differ from the rate measured with the extensometer. Thus, the nominal strain rate is just an indicator, only measurement from the extensometer were used for the purpose of the identification and/or the experimental validation. It should be mentioned that all the mechanical tests were performed within a moderately slow strain rate range (between $1.10^{-3}$ and $5 \cdot 10^{-2} \mathrm{~s}^{-1}$ ), at which the selfheating phenomenon remains relatively limited. Thus, it can be reasonably assumed that the material stays under approximately isothermal conditions. 


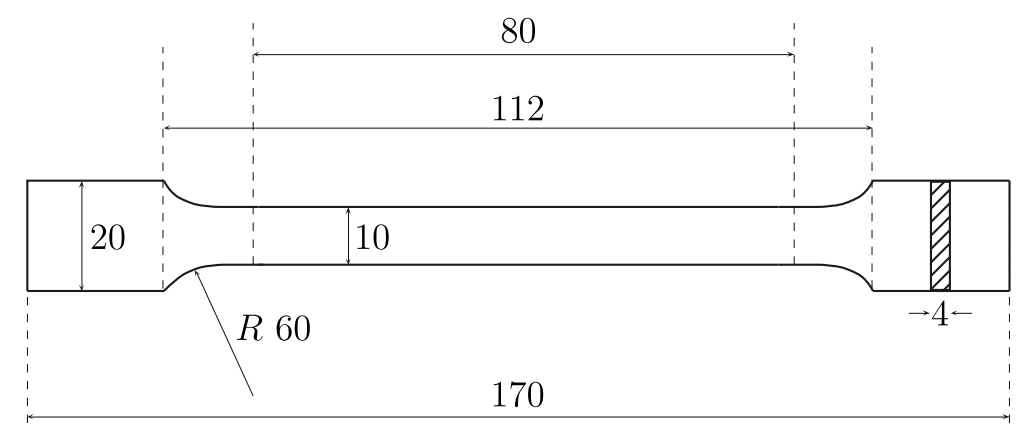

Figure 3: ISO527-2-1A tensile specimen (dimensions in mm).

In order to capture accurately all the accounted mechanisms, the following experimental program is proposed. It involves several types of tests:

- Monotonic tensile tests, noted hereafter as "M" (Figure 4a), performed at different strain rates emphasizing the global rate effect due to viscoelasticity and viscoplasticity. In the present case, the following nominal strain rates are chosen: $5.10^{-3}, 1.10^{-2}$ and $5.10^{-2} \mathrm{~s}^{-1}$.

- Incremental Load/Unload tensile tests, noted hereafter as "LU" (Figure 4b), performed at different strain rates. Besides highlighting the global rate effect, these tests bring out the apparent stiffness reduction due to damage, the inelastic strains caused by the viscoplasticity and the hysteresis loops that are induced by the viscoelasticity acting under short characteristic times. In the present case, the following nominal strain rates are chosen: $1.10^{-3}, 5.10^{-3}, 1.10^{-2}$ and $5.10^{-2} \mathrm{~s}^{-1}$

- Incremental Load/Relaxation/Unload/Relaxation tensile tests, noted hereafter as "LRUR" (Figure 4c), performed at one strain rate. This test allows to emphasized the relaxation occurring on a long term caused by the viscoelasticity acting under long characteristic times. 
In the present case, a strain rate of $1.10^{-2} \mathrm{~s}^{-1}$ is chosen. As shown on Figure 4c, the "LRUR" test involves a certain time during which the strain is held constant, longer is this holding time, better the long term relaxation will be emphasized. In the present case, a holding time of $60 \mathrm{~s}$ is considered.

- Cyclic tensile test, noted hereafter as "C" (Figure 4d), performed at the rate of $1.10^{-2} \mathrm{~s}^{-1}$. This test is mainly kept for the validation of model under a cyclic loading condition.

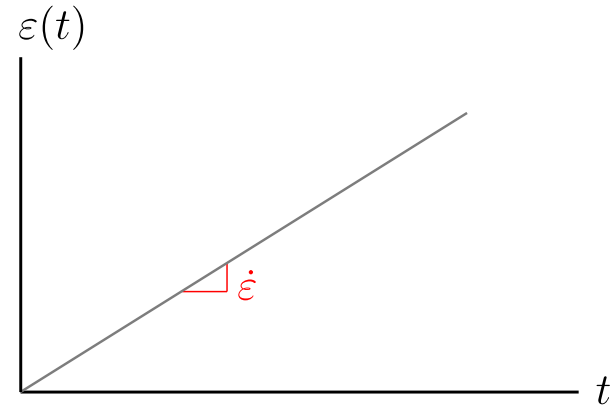

(a) Monotonic "M"

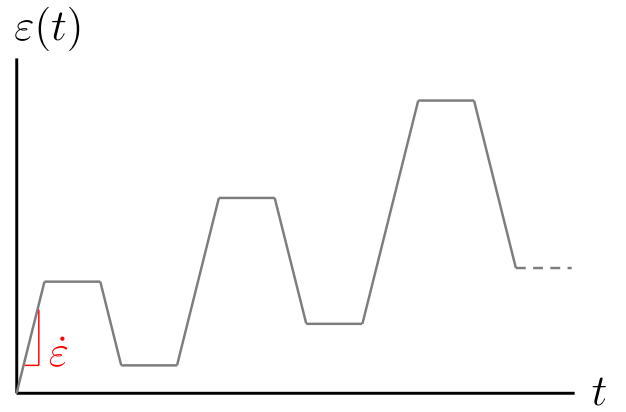

(c) Load/Relaxation/Unload/Relaxation "LRUR"

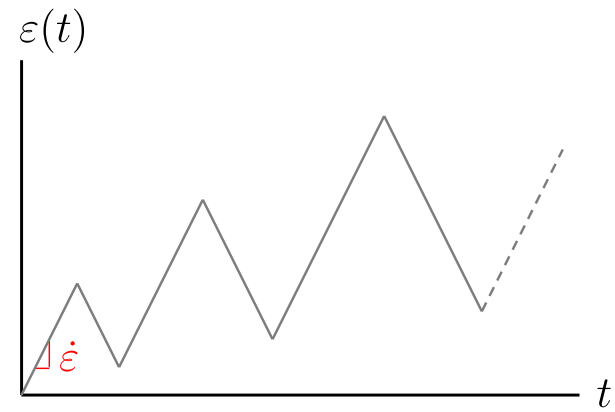

(b) Load/Unload "LU"

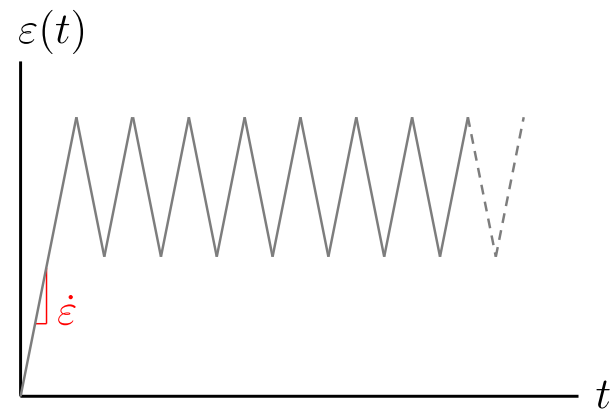

(d) Cyclic "C"

Figure 4: Performed strain controlled test configurations. 


\subsection{Identification strategy}

It is well known that parameters related to viscoelasticity can be identified from Dynamic Mechanical Analysis (DMA) by calibrating the storage and loss modulus $E^{\prime}$ and $E^{\prime \prime}$ on the master curve within a predefined frequency range (Lakes, 1999). Nevertheless, even though from a theoretical point of view, this method is very convenient, it may not provide satisfying results. Indeed, these tests are usually performed at very low stress levels which may not cause the same mechanisms than those considered in the developed model. In the present work, a direct identification procedure is proposed as an alternative way.

The identification of such complex constitutive laws, where several mechanisms are simultaneously involved, often requires a suitable methodology. Indeed, most of the times it is impossible to completely isolate a single mechanism in order to identify independently its related parameters. Consequently, the identification procedure necessitates the use of reverse engineering methods. To do so, an optimization algorithm based on the Levenberg-Marquardt technique (Meraghni et al., 2011, 2014) has been used. The aim of this method is to identify a set of parameters $\boldsymbol{p}$ by minimizing a cost function $C$. In the present case, this cost function is expressed by the least squares between the numerical and experimental uni-axial stress responses, $\sigma^{\text {num }}$ and $\sigma^{e x p}$, respectively:

$$
C(\boldsymbol{p})=\frac{1}{2} \sum_{k=1}^{N_{p}} w_{k}\left[\sigma_{k}^{n u m}(\boldsymbol{p})-\sigma_{k}^{e x p}\right]^{2},
$$

where $N_{p}$ denotes the number of measuring points and $w_{k}$ an eventual weight 
given to each of them. However, in order to ensure the robustness of the identification procedure, it is strongly advised to avoid identifying all the parameters simultaneously. Instead, the following three steps strategy is applied:

- Step 1: At low stress levels, the effects of damage and viscoplasticity can be neglected and then the material remains in the viscoelastic regime. This condition can be assumed to be true within the first load/unload cycle of the "LU" tests. Thus, at the first step the parameters related to viscoelasticity are assessed from these data.

- Step 2 : Next, the parameters previously identified are kept constant while the parameters related to viscoplasticity and damage are optimized from the "M" tests and two of the four "LU" tests, preferentially the slowest and the fastest.

- Step 3 : Finally, starting from the previously obtained values, all the parameters are optimized once more, from the same tests than the previous step and the "LRUR" test in order to adjust the relaxation effects occurring on the long term.

This procedure is summarised in Table 2 . 
Table 2: Summary of the identification procedure.

\begin{tabular}{|c|c|c|c|c|c|}
\hline Nominal & "M" & \multicolumn{2}{|c|}{ "LU" } & "LRUR" & "C" \\
strain rate & & $1^{\text {st }}$ cycle & complete & & \\
\hline $1.10^{-3} \mathrm{~s}^{-1}$ & - & Step 1 & Steps 2, 3 & - & - \\
\hline $5.10^{-3} \mathrm{~s}^{-1}$ & Steps 2, 3 & Step 1 & validation & - & - \\
\hline $1.10^{-2} \mathrm{~s}^{-1}$ & Steps 2, 3 & Step 1 & validation & Step 3 & validation \\
\hline $5.10^{-2} \mathrm{~s}^{-1}$ & Steps 2,3 & Step 1 & Steps 2, 3 & - & - \\
\hline
\end{tabular}

Note that, except the Poisson ratio $\nu$, all the other parameters of the model can be identified from a purely uni-axial stress-strain response. Obviously, the Poisson ratio can be obtained from measurements of the transversal strain in addition to the longitudinal one, at a stress level low enough so that no plastic strains are generated.

\subsection{Identification and experimental validation}

Using the previously described identification strategy, the parameters governing the proposed constitutive model have been identified considering four Kelvin-Voigt branches for the viscoelastic part besides the viscoplaticity coupled to the damage. The values of the parameters are listed in Table 3. 
Table 3: Identified parameters for polyamide $(6,6)$.

\begin{tabular}{|c|c|c|c|c|}
\hline \multicolumn{2}{|l|}{ Mechanical feature } & \multirow{2}{*}{$\begin{array}{l}\text { Parameter } \\
E_{e}\end{array}$} & \multirow{2}{*}{$\frac{\text { value }}{2320}$} & \multirow{2}{*}{$\frac{\text { unit }}{\mathrm{MPa}}$} \\
\hline Viscoelasticity & Single spring & & & \\
\hline & Kelvin-Voigt branch 1 & $E_{v_{1}}$ & 8648 & $\mathrm{MPa}$ \\
\hline & & $\eta_{v_{1}}$ & 4442 & MPa.s \\
\hline & & $\tau_{v_{1}}=\frac{\eta_{v_{1}}}{E_{v_{1}}}$ & 0.51 & $\mathrm{~s}$ \\
\hline & Kelvin-Voigt branch 2 & $E_{v_{2}}$ & 13884 & $\mathrm{MPa}$ \\
\hline & & $\eta_{v_{2}}$ & 457955 & MPa.s \\
\hline & & $\tau_{v_{2}}=\frac{\eta_{v_{2}}}{E_{v_{2}}}$ & 32.98 & $\mathrm{~S}$ \\
\hline & Kelvin-Voigt branch 3 & $E_{v_{3}}$ & 4491 & $\mathrm{MPa}$ \\
\hline & & $\eta_{v_{3}}$ & 169048 & MPa.s \\
\hline & & $\tau_{v_{3}}=\frac{\eta_{v_{3}}}{E_{v_{3}}}$ & 37.64 & $\mathrm{~s}$ \\
\hline & Kelvin-Voigt branch 4 & $E_{v_{4}}$ & 12725 & $\mathrm{MPa}$ \\
\hline & & $\eta_{v_{4}}$ & 1307441 & MPa.s \\
\hline & & $\tau_{v_{4}}=\frac{\eta_{v_{4}}}{E_{v_{4}}}$ & 102.75 & $\mathrm{~s}$ \\
\hline \multirow{7}{*}{$\begin{array}{l}\text { Viscoplasticity } \\
\text { coupled to damage }\end{array}$} & Yield threshold & $R_{0}$ & 1.01 & $\mathrm{MPa}$ \\
\hline & Hardening function & $K$ & 1456.85 & $\mathrm{MPa}$ \\
\hline & & $n$ & 0.585 & - \\
\hline & Viscous stress function & $H$ & 49.13 & ${\mathrm{MPa} . \mathrm{S}^{m}}^{m}$ \\
\hline & & $m$ & 0.056 & - \\
\hline & Damage & $S$ & 14.423 & $\mathrm{MPa}$ \\
\hline & & $\beta$ & -1.656 & - \\
\hline
\end{tabular}


Figures 5, 6, 7 and 8 show the comparison between the simulated curves and the experimental data used for the identification. The obtained results demonstrate the model's capability to capture properly the rate effect, the stiffness reduction as well as the long term relaxation. However, the short term relaxation appears to be less accurate as the wideness of the hysteresis loops is not always well captured, especially when the level of strain/stress becomes high. This issue might be resolved by considering more Kelvin-Voigt branches or the use of non-linear viscoelasticity (Khan et al., 2006), which could allow a better description of the viscoelastic mechanisms occurring on the short term. The additional Kelvin-Voigt branches though require a significant increase on the number of material parameters.

Figures 9, 10 and 11 show the comparison between the simulated curves and experimental data that has not been used for the identification. The good agreement with the experiments validates the previously identified parameters and then confirms the prediction capabilities of the model.

On the slowest and fastest "LU" tests (Figures 6 and 7) that were used for the identification, it can be noticed that the accuracy of the model predictions, in terms of stress, decrease for high strain levels. Nevertheless, for the "LU" test performed at intermediate strain rates (Figures 9, 10) this issue does not appear any more. This point demonstrates the limitations of the model regarding its strain rate range validity. 


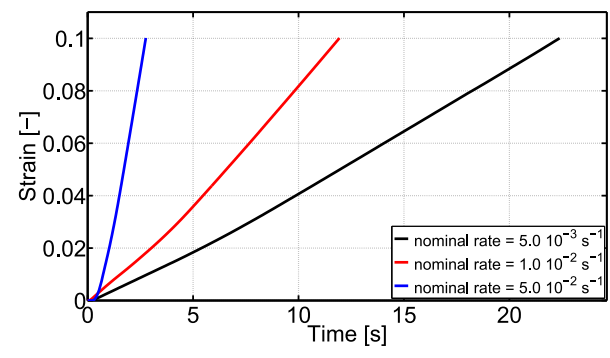

(a) Applied strain vs. time

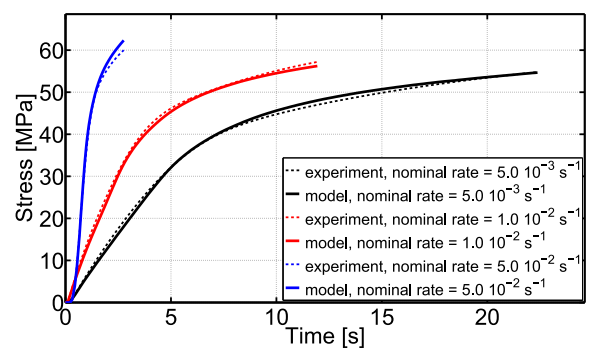

(b) Stress response vs. time

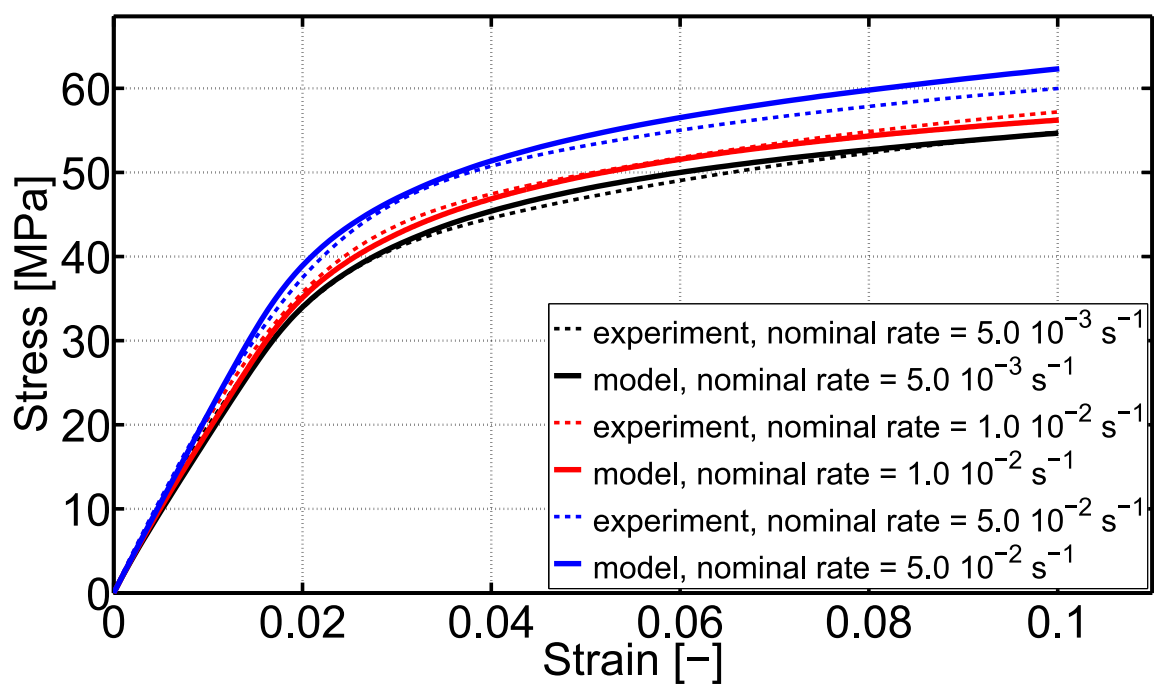

(c) Stress vs. strain

Figure 5: Monotonic "M" tensile tests for different strain rates (data used for the identification), comparison between model and experiments. 


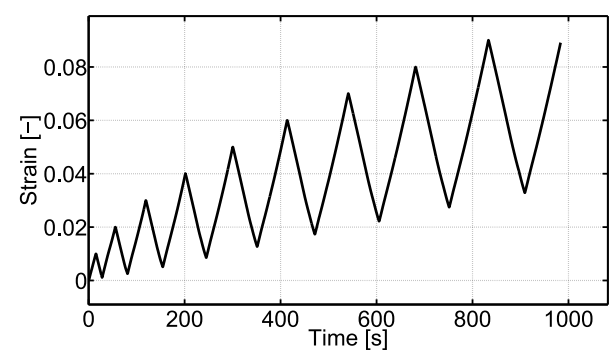

(a) Applied strain vs. time

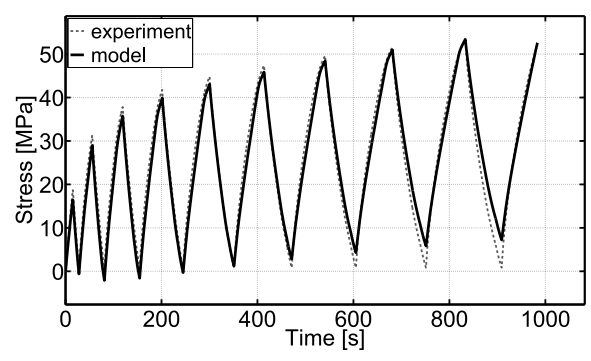

(b) Stress response vs. time

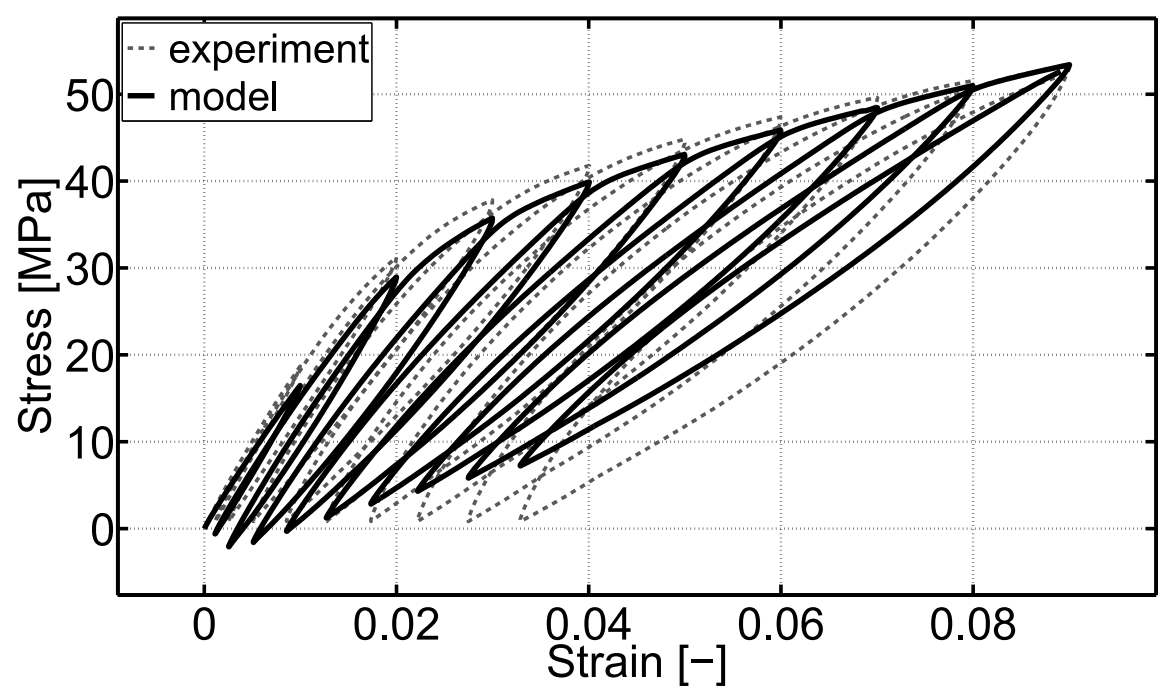

(c) Stress vs. strain

Figure 6: Load/Unload "LU" tensile at the nominal strain rate of $1.010^{-3} \mathrm{~s}^{-1}$ (data used for the identification), comparison between model and experiments. 


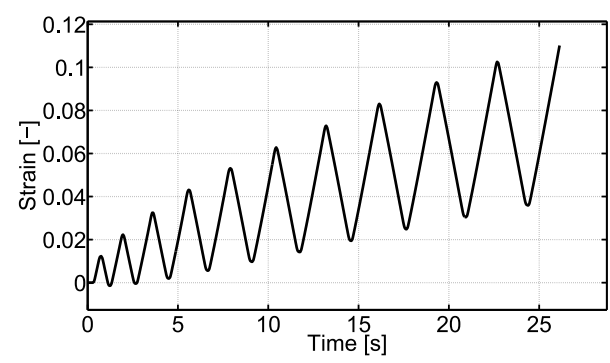

(a) Applied strain vs. time

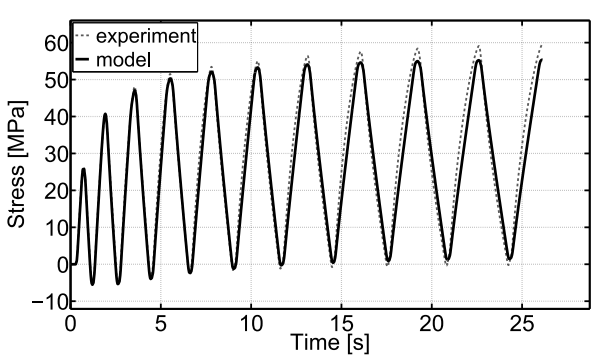

(b) Stress response vs. time

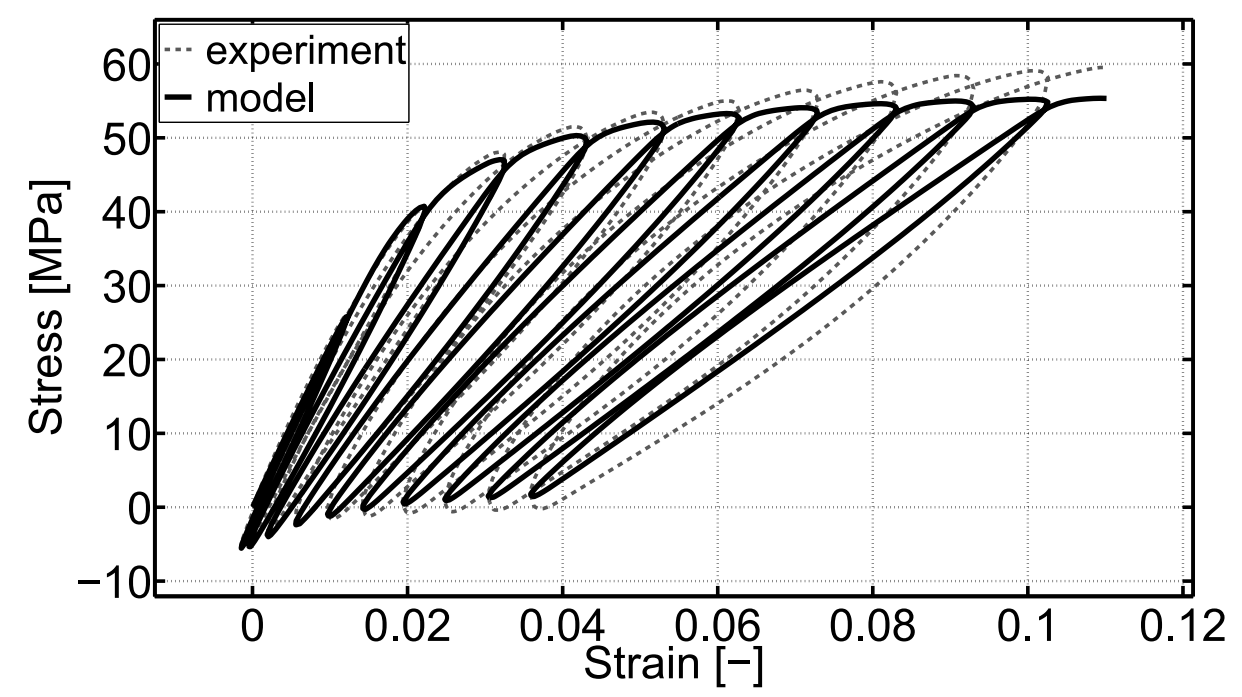

(c) Stress vs. strain

Figure 7: Load/Unload "LU" tensile at the nominal strain rate of $5.010^{-2} \mathrm{~s}^{-1}$ (data used for the identification), comparison between model and experiments. 


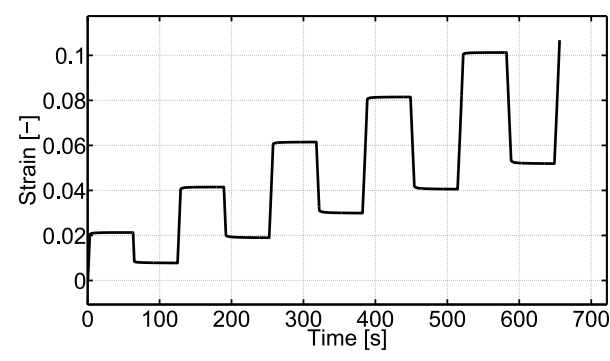

(a) Applied strain vs. time

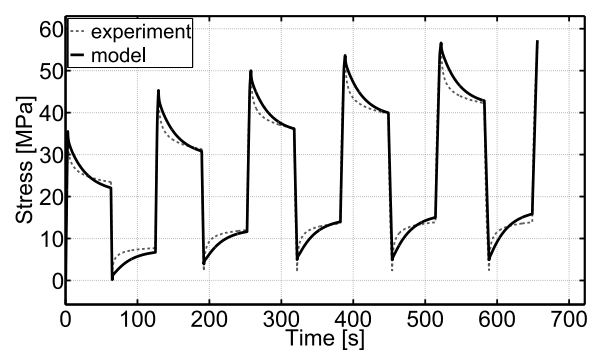

(b) Stress response vs. time

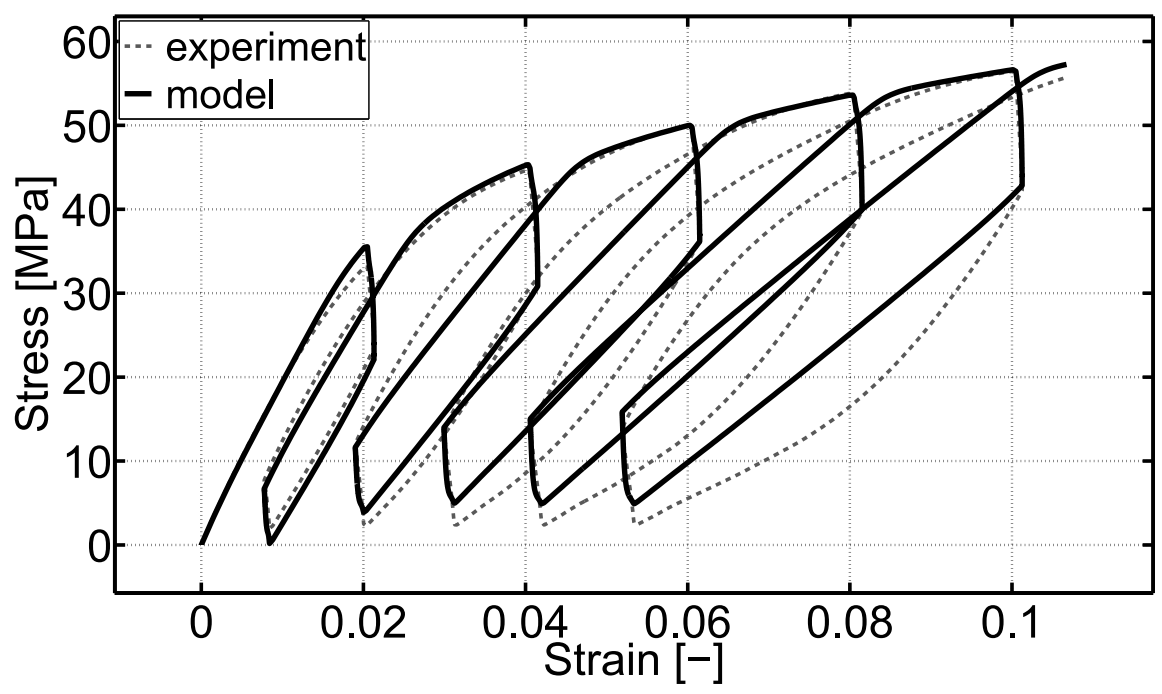

(c) Stress vs. strain

Figure 8: Load/Relaxation/Unload/Relaxation "LRUR" tensile test (data used for the identification), comparison between model and experiments. 


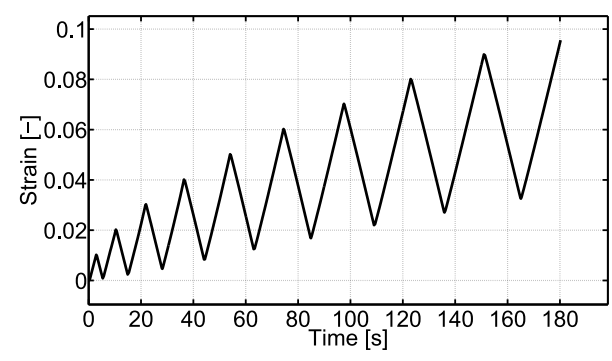

(a) Applied strain vs. time

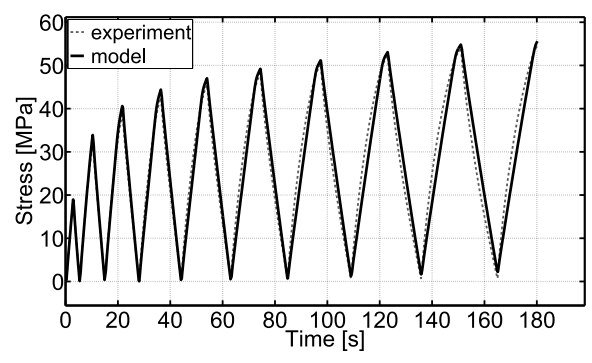

(b) Stress response vs. time

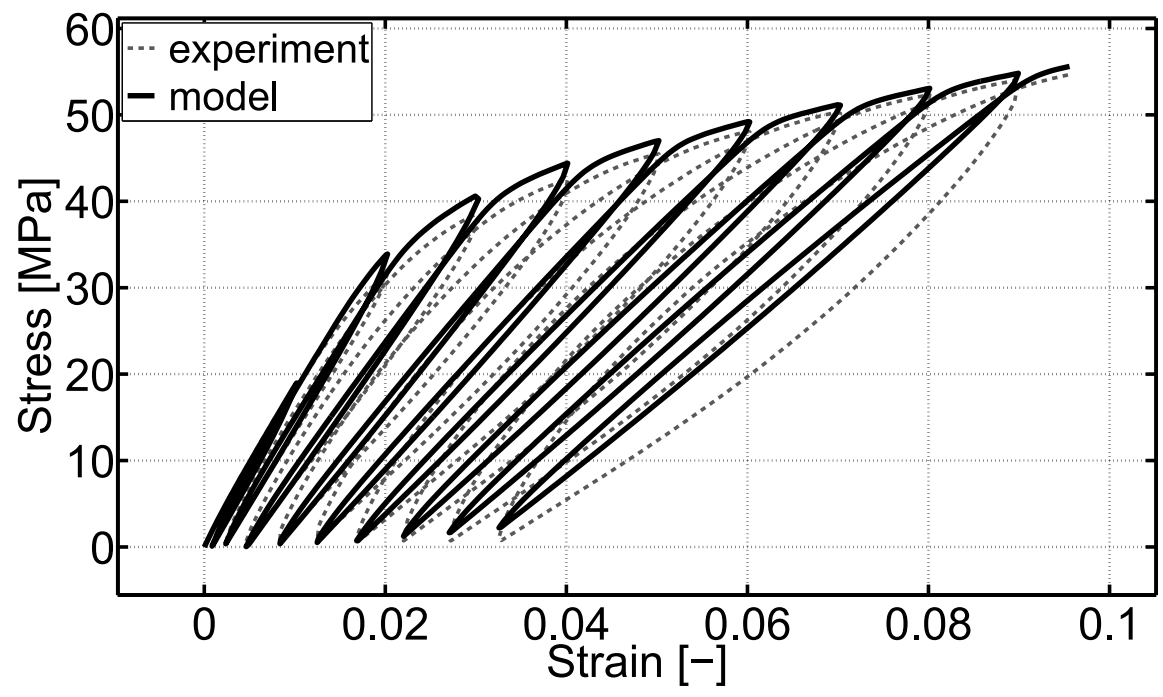

(c) Stress vs. strain

Figure 9: Load/Unload "LU" tensile at the nominal strain rate of $5.010^{-3} \mathrm{~s}^{-1}$ (validation), comparison between model and experiments. 


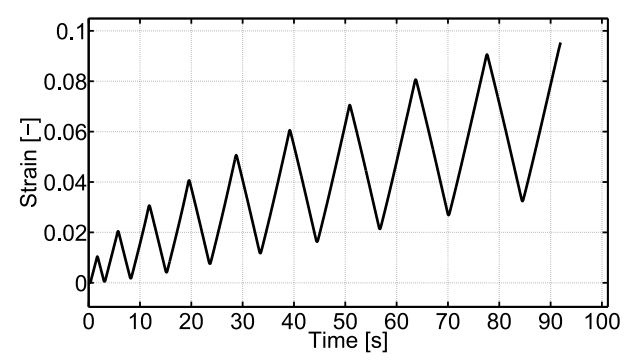

(a) Applied strain vs. time

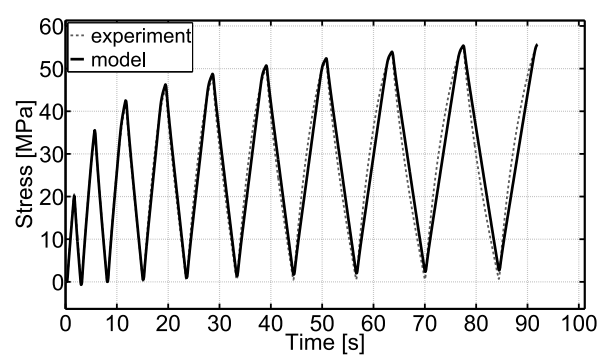

(b) Stress response vs. time

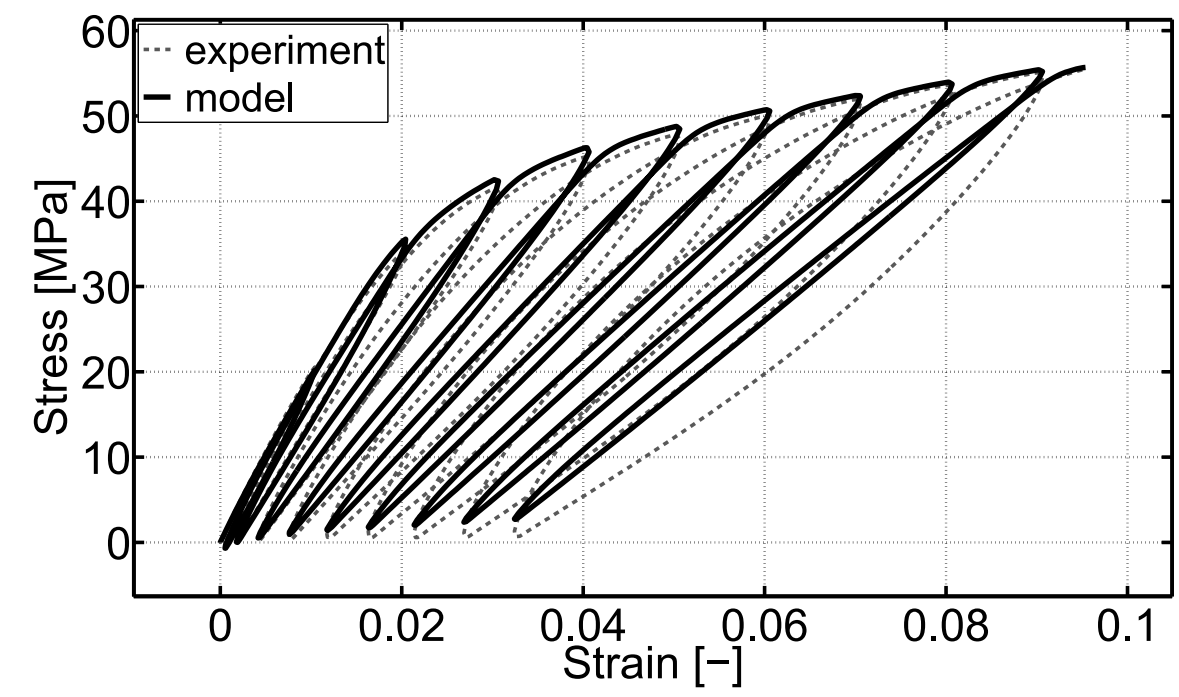

(c) Stress vs. strain

Figure 10: Load/Unload "LU" tensile at the nominal strain rate of $1.010^{-2} \mathrm{~s}^{-1}$ (validation), comparison between model and experiments. 


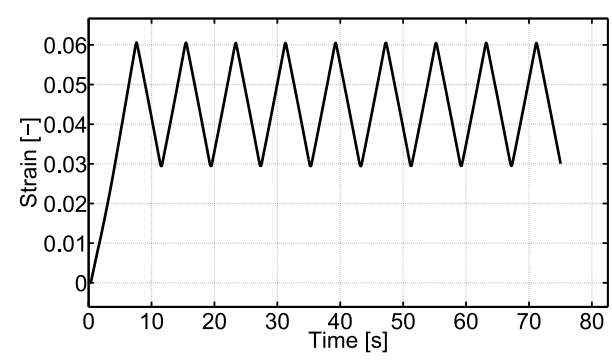

(a) Applied strain vs. time

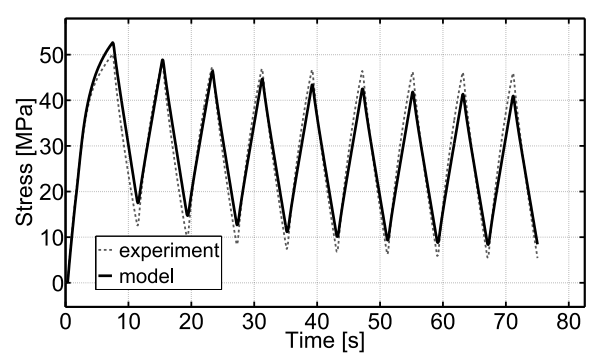

(b) Stress response vs. time

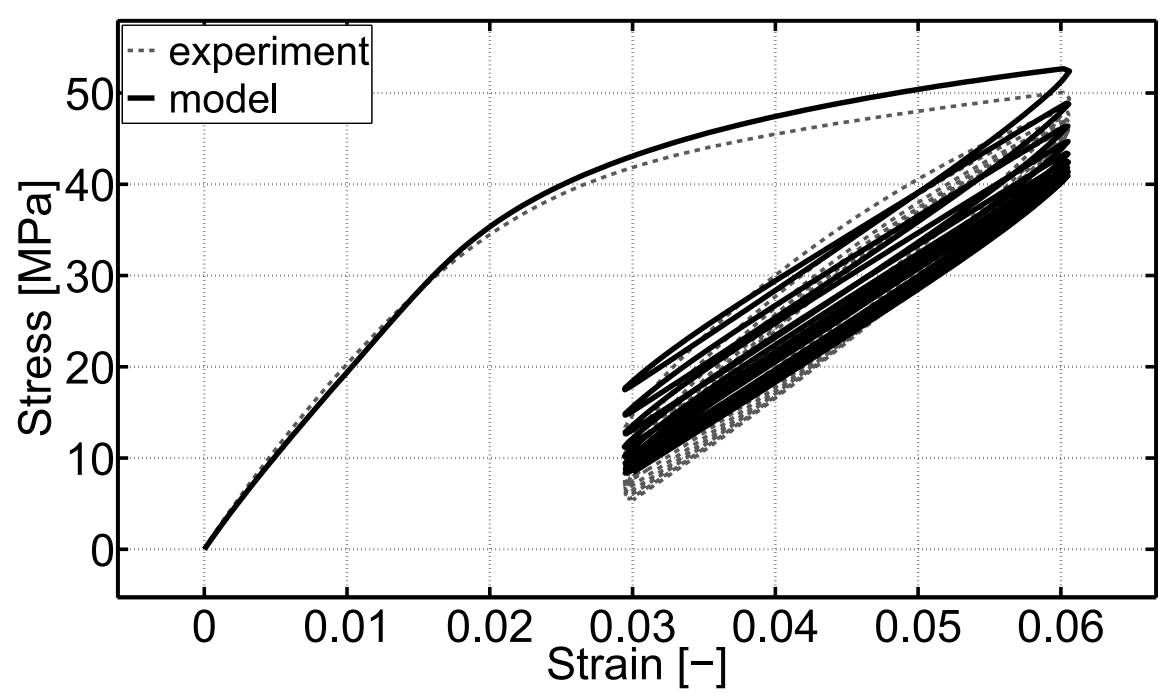

(c) Stress vs. strain

Figure 11: Cyclic "C" tensile test (validation), comparison between model and experiments.

\section{Numerical simulations and dissipative behaviour}

To provide a better understanding regarding the dissipative behaviour of the proposed constitutive model, three uni-axial simulations have been performed with the previously identified set of parameters (Table 3):

- A cyclic strain controlled simulation in which a uni-axial strain of 0.05 
is applied in $5 \mathrm{~s}$ before returning to zero strain in $5 \mathrm{~s}$, this cycle is repeated 10 times (Figure 12).

- A cyclic stress controlled simulation in which a uni-axial stress of 50 $\mathrm{MPa}$ is applied in $5 \mathrm{~s}$ before returning to zero stress in $5 \mathrm{~s}$, this cycle is repeated 10 times (Figure 13).

- A mixed strain/stress controlled simulation in which a strain of 0.05 is first applied in $5 \mathrm{~s}$, this strain is then held during $200 \mathrm{~s}$. Next, the material is unloaded to zero stress in $5 \mathrm{~s}$ and the stress is then kept to zero during $200 \mathrm{~s}$ (Figure 14).

The results of these simulations (Figures 12, 13 and 14) highlight well the capabilities of the proposed model for capturing various mechanisms that occur on several ranges of time.

When a strain controlled cyclic tension is applied (Figure 12a), then the resulting stress exhibits a global relaxation throughout the cycling, as the peak stress decreases from one cycle to another (Figure 12b). Similarly, when stress control cyclic tension is applied (Figure 13b), then the resulting strain exhibits a global creep throughout the cycling, as the peak strain increases from one cycle to another (Figure 13a). Those effects are clearly due to the Kelvin-Voigt branches with a long characteristic time that actually move on a range of time that is far beyond the time length of a single cycle (Figures 12c and 13c). Regarding the evolution of the viscoplastic strain and the damage, when a cyclic strain is applied, most of the evolution occurs during the first cycle, while it remains almost null during the following cy- 
cles (Figure 12c and 12d). A relatively different behaviour can be observed when a cyclic stress is applied. Indeed, in this case, more viscoplastic strain and damage are generated during the beginning of the cycling (Figure 13c and 13d). This evolution gradually decreases from one cycle to another and seems to stabilize after a certain number of cycles.

In term of energy balance (Figures 12f, $13 \mathrm{f}$ and 14f), it can be remarked first that the strain energy $W_{\varepsilon}$ is well equal to the sum of the stored and dissipated energy, $\rho \psi$ and $\Phi$ respectively. Moreover the dissipated energy is always increasing that is well in accordance with the Clausius Duhem inequality (14).

The third simulation (Figure 14) highlight well the dissipative behaviour of the material (Figure 14f). Indeed, during the first phase of the simulation, when the material is loaded (strain control) and most of the dissipation is caused by the viscoplastic and the damage mechanisms (Figures 14c and 14d). Next, when the strain is held constant, the material is then relaxing as the stress is decreasing (Figure 14b), during this phase, the material exhibits a energy transfer, the strain energy does not evolve while the stored energy is converted into dissipated energy under the action of the viscoelastic mechanisms (Figures 14c and 14f). The material is next unloaded (stress control), then a part of the stored energy is quasi-instantaneously recovered (Figure 14f). Finally, the stress is kept to zero, during this phase, the material is creeping as strain is decreasing, another part of the stored energy is recovered with a retard effect and is converted into dissipated energy (Figure 14f). 
When all the Kelvin-Voigt branches have recovered their strain, only the viscoplastic strain remains with its related stored energy that is irrecoverable, as explained in Section 2.2.2.

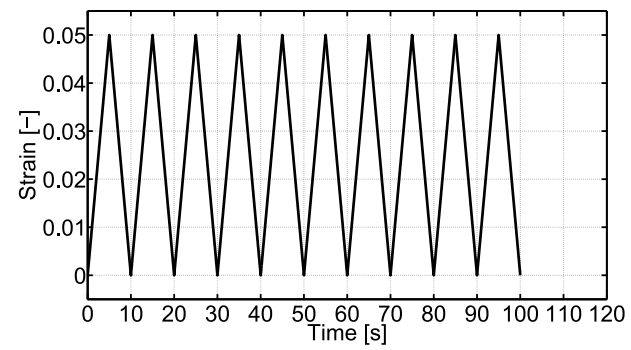

(a) Applied strain vs. time

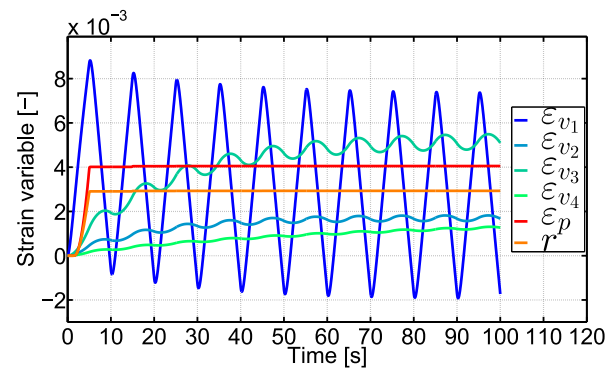

(c) Strain internal state variables vs. time

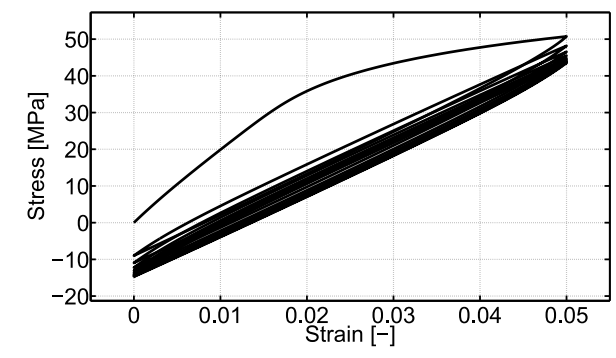

(e) Stress vs. strain

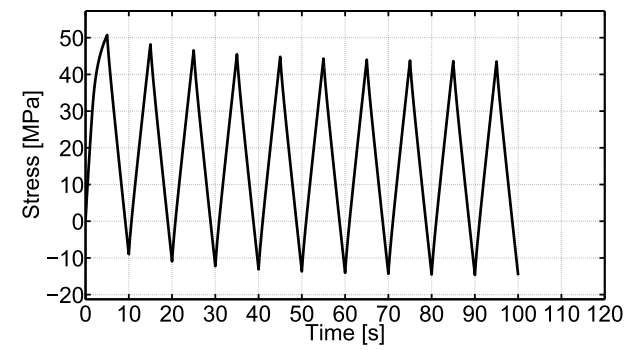

(b) Stress response vs. time

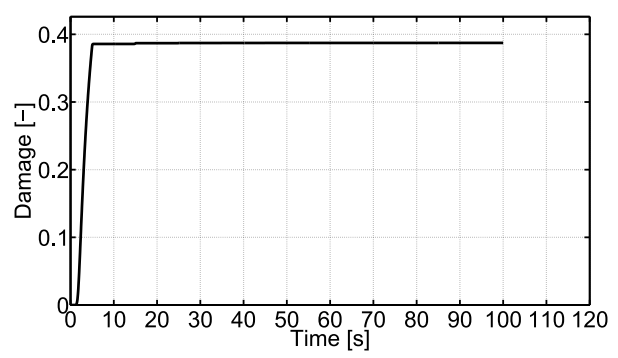

(d) Damage vs. time

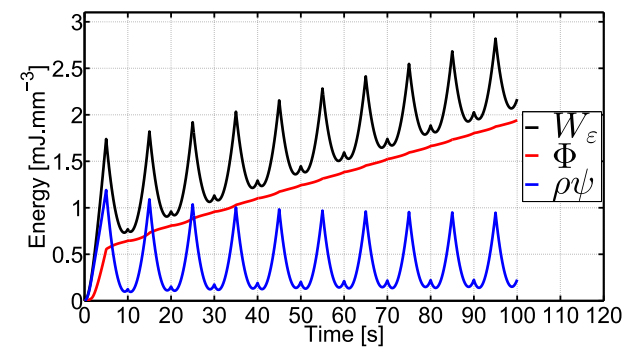

(f) Strain $\left(W_{\varepsilon}\right)$, dissipated $(\Phi)$ and stored $(\rho \psi)$ energies vs. time

Figure 12: Uni-axial strain controlled cyclic simulation. 


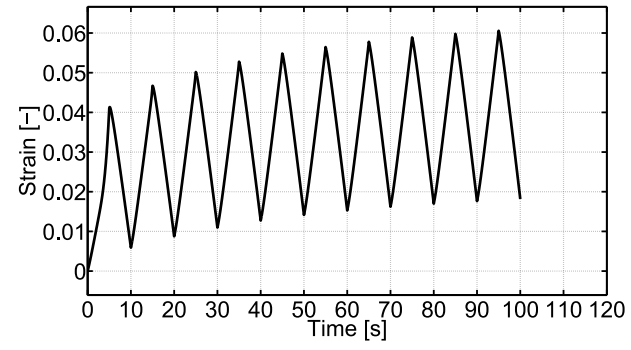

(a) Strain response vs. time

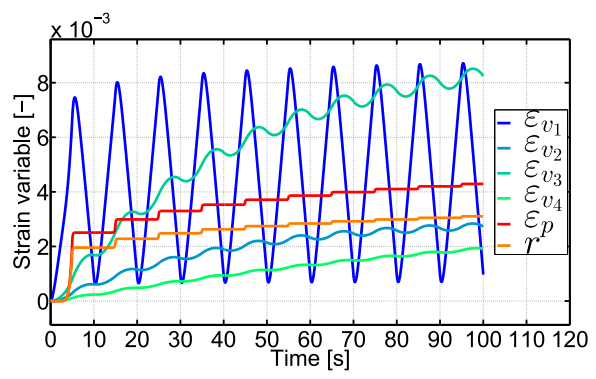

(c) Strain internal state variables vs. time

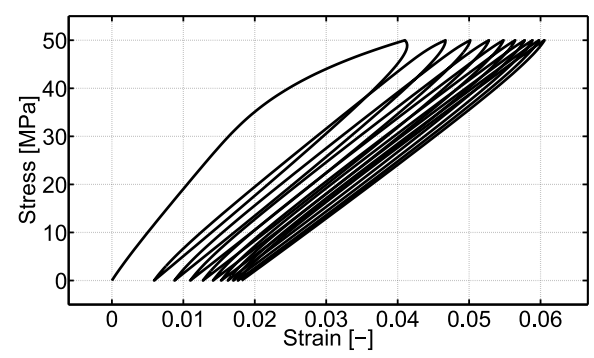

(e) Stress vs. strain

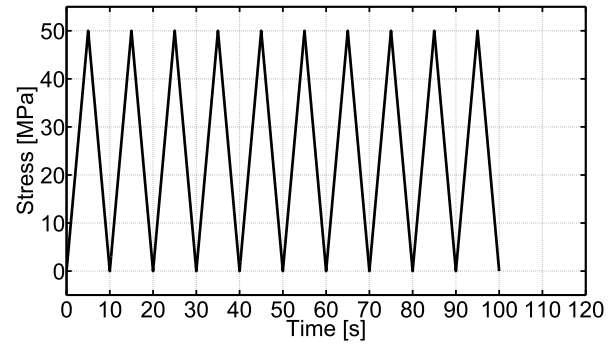

(b) Applied stress vs. time

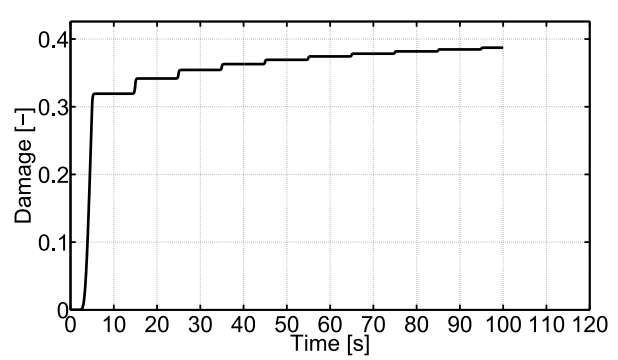

(d) Damage vs. time

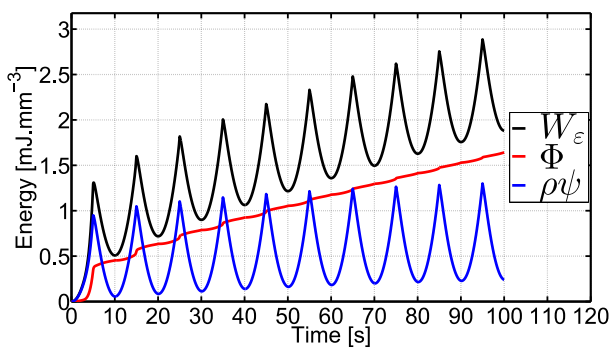

(f) Strain $\left(W_{\varepsilon}\right)$, dissipated $(\Phi)$ and stored $(\rho \psi)$ energies vs. time

Figure 13: Uni-axial stress controlled cyclic simulation. 


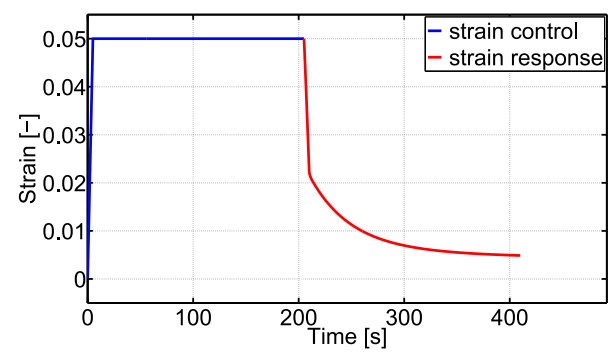

(a) Strain vs. time

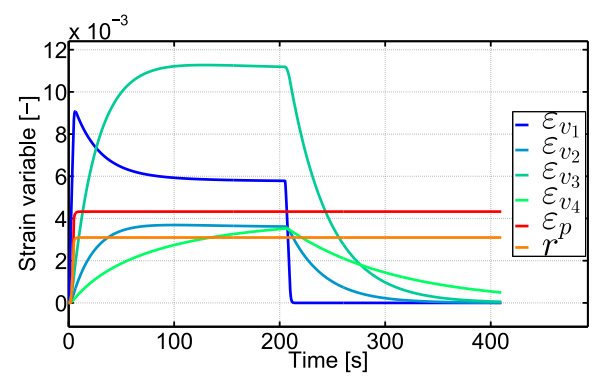

(c) Strain internal state variables vs. time

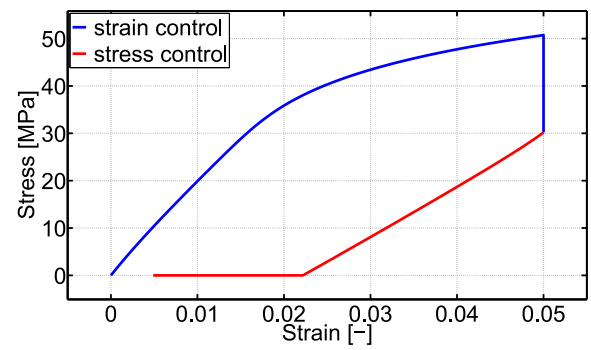

(e) Stress vs. strain

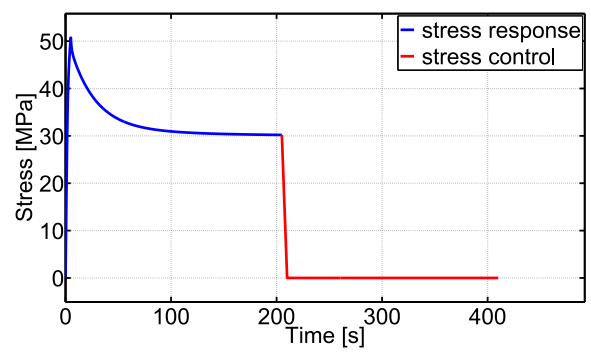

(b) Stress vs. time

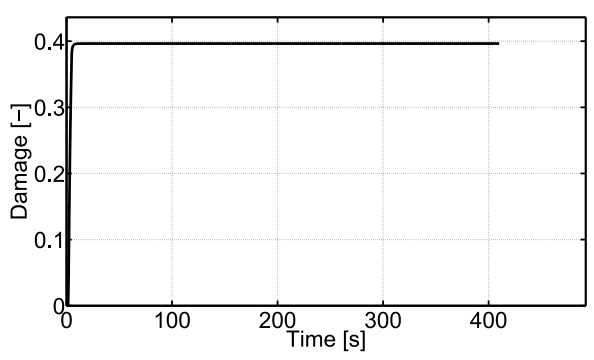

(d) Damage vs. time

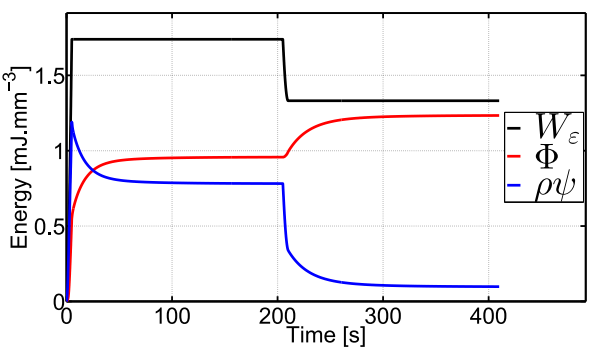

(f) Strain $\left(W_{\varepsilon}\right)$, dissipated $(\Phi)$ and stored $(\rho \psi)$ energies vs. time

Figure 14: Simulation of a uni-axial strain controlled tension relaxation followed by a stress controlled unload and strain recovery. 


\section{Structural FE application and model capabilities}

In order to demonstrate the model capabilities in simulating the behaviour of a 3D structure, the present section deals with an example of $\mathrm{FE}$ analysis performed on a bracket (Figure 15a) made of polyamide $(6,6)$ with the previously identified properties (Table 3) and a Poisson ration of 0.3 . The bottom face of the bracket is clamped while a cyclic loading along the $x$ direction is applied on the holes located at the top (Figure 15c). This load is set up on a node that lies at the centre of the hole and is linked to it using a tie constrain. Due to symmetry, half of the structure is represented and appropriate boundary conditions are accounted on the plane of symmetry (Figure 15b). The whole geometry is meshed with quadratic tetrahedron elements (C3D10) as shown on Figure 15b. A cycle of loading is defined in 5 steps: first $-5 \mathrm{kN}$ is applied in $5 \mathrm{~s}$ on the bracket. Next, half of the load is released in $2.5 \mathrm{~s}$ and the remaining load is held for another $2.5 \mathrm{~s}$. Then, the structure is totally unloaded in $2.5 \mathrm{~s}$ and the bracket is kept free of loading during another 2.5 s. This cycle is repeated 10 times (Figure 16a). The computation is carried out with a constant time increment of $0.1 \mathrm{~s}$. 


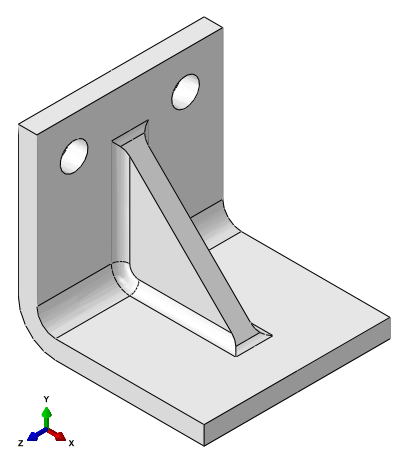

(a) Bracket

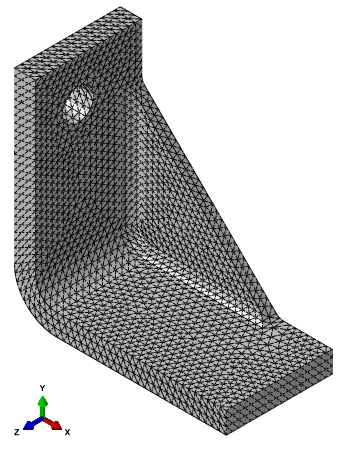

(b) Meshed bracket, half of the structure is represented due to symmetry

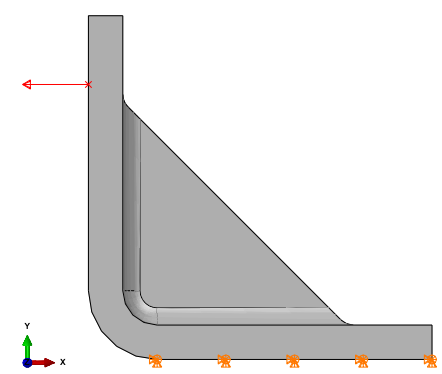

(c) Loading and boundary conditions applied on the bracket

Figure 15: Example of FE analysis.

The proposed implicit implementation shows a good efficiency as for each time increment only one or two iterations are necessary to reach the global convergence. The obtained results well transcribe the temporal behaviour of the proposed model at the scale of the structure. Indeed, it can be seen that the whole bracket is creeping on several time ranges. Creep effects can be observed during the steps where the load is held among a single cycle but also globally throughout the cycling as the peak of displacement is increasing from one cycle to another (Figure 16b). 


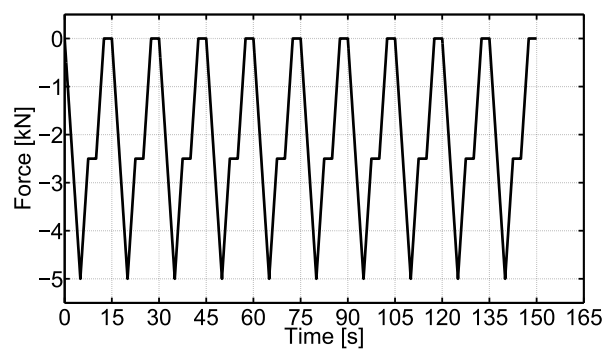

(a) Applied force along the $x$ axis

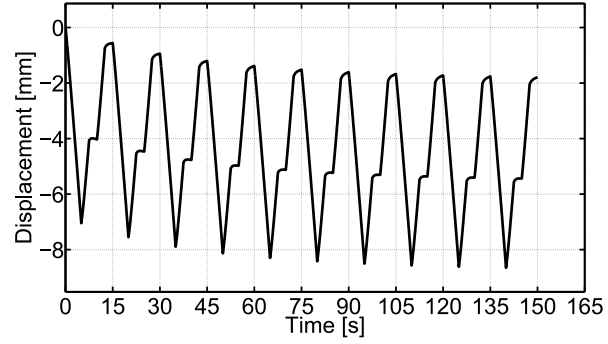

(b) Resulting displacement along the $x$ axis

Figure 16: Applied force and resulting displacement at the node lying at the centre of the hole.

The damage effects are also accounted at the scale of the structure. The model is able to capture the initiation of the damage on the most stressed point of the bracket and then is gradually spreading around this point during the cycling (Figure 17a). As remarked on the uni-axial simulation (Figure 13d), damage tends to stabilize after a certain number of cycles (Figure 17b). 

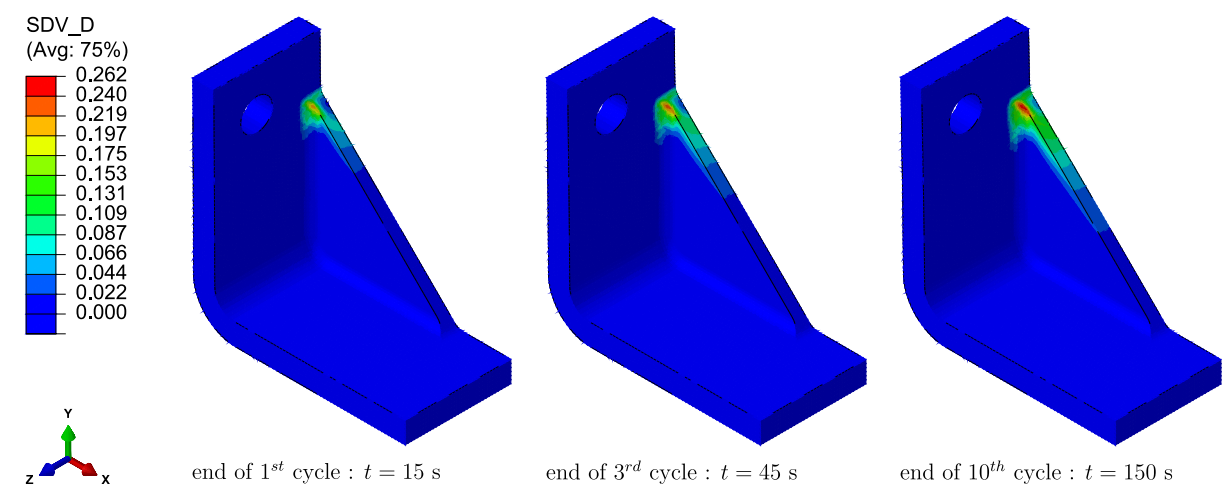

(a) Damage distribution within the structure (contour plot)

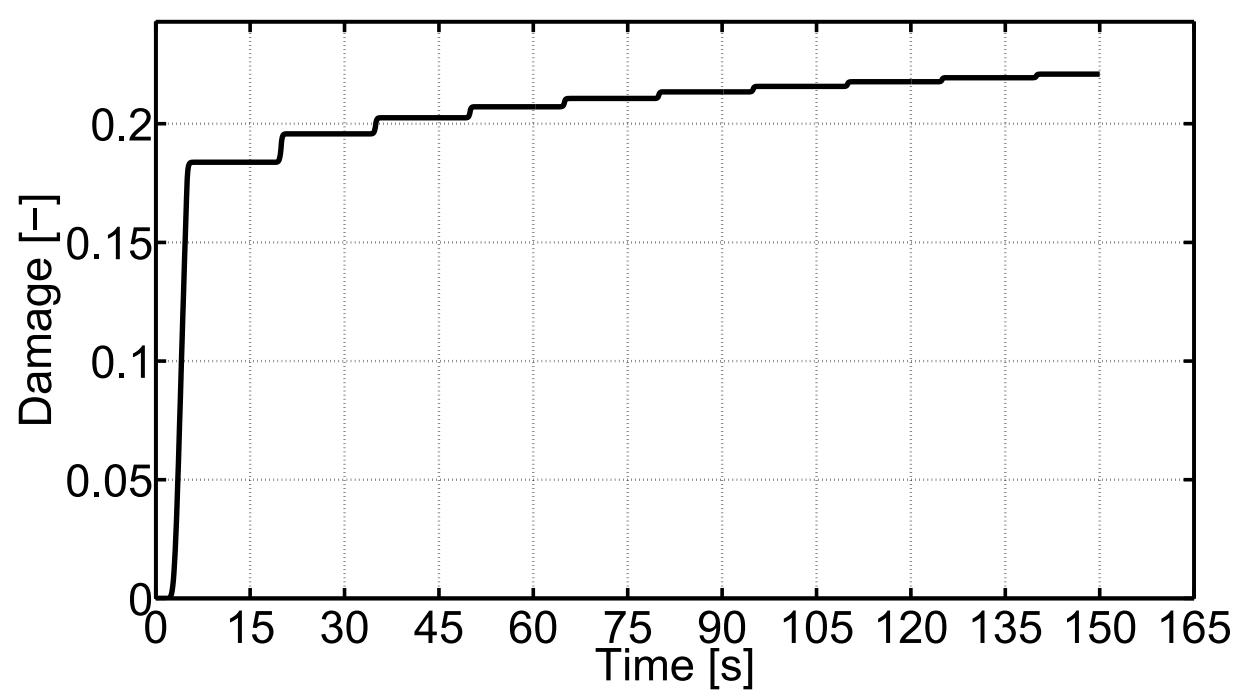

(b) Damage vs. time at the most stressed integration point

Figure 17: Evolution and repartition of damage.

\section{Conclusions and perspectives}

In this paper, a constitutive model for thermoplastic polymers has been proposed along with a proper thermodynamical formalism and its implicit numerical implementation, accounting for viscoelastic, viscoplastic and damage mechanisms. The multiple Kelvin-Voigt branches in the viscoelastic part 
of the free energy potential is essential for identifying the viscous behaviour of the polymer under an extended range of loading frequency/rate. A set of experimental tests has been carried out according to different loading configurations on the polyamide (6.6), allowing to successfully identify the parameters of the model through a stepwise strategy. The good agreement between experimental and numerical results demonstrates the capabilities of the model to capture the mechanical behaviour driven by a multi-mechanisms coupled response with a single set of parameters. Complementary simulations under complex loading have been performed in order to provide a better understanding of the dissipative behaviour of the material when subjected to cyclic loading, relaxation and creep. The formulated model allows to identify the influence of each nonlinear mechanism (viscoelasticity, viscoplasticity and damage) in the overall material response under complex and non-proportional loading paths. Finally, the model has been implemented into a FE code towards performing a 3D structural analysis.

In terms of perspectives, the current work can be extended in three different directions: i) The thermodynamical framework allows the model to be easily extended to fully coupled thermomechanical analyses. Indeed, because the thermomechanical interactions are known to be important in thermoplastic polymers (Benaarbia et al., 2014, 2015). Such a coupling requires the integration of the self-heating generated by the dissipation and the thermomechanical coupling sources (Ovalle Rodas et al., 2014, 2016; Benaarbia et al., 2015; Chatzigeorgiou et al., 2016; Yu et al., 2017a,b). ii) The present constitutive equations can be used for the modelling of thermoplastic based 
composites through a micromechanical scale transition scheme, whether by mean field (Lagoudas et al., 1991; Chaboche et al., 2005; Miled et al., 2013) or full field theories (Chatzigeorgiou et al., 2015, 2016). Indeed, in reinforced thermoplastics, the rheological behaviour of the matrix is known to have coupled effects with the damage mechanisms that occur at the scale of the microstructure (Fitoussi et al., 2013; Arif et al., 2014a,b) and can then be accounted for using such approaches. iii) The model, integrating temporal effects on several time ranges, can provide a basis for modelling high cycle fatigue. Of course, such fatigue analyses require model reduction techniques (Cojocaru and Karlsson, 2006; Haouala and Doghri, 2015) that enable simulations on long time periods, as well as advanced fatigue theories for polymers (Nouri et al., 2009; Launay et al., 2013b).

\section{Acknowledgements}

This paper is part of the COPERSIM project managed by IRT Jules Verne (French Institute in Research and Technology in Advanced Manufacturing Technologies for Composite, Metallic and Hybrid Structures). The authors wish to associate the industrial and academic partners of this project;

respectively Arts et Metiers ParisTech, Solvay, Plastic Omnium, PSA and Renault.

\section{Appendix A. Expressions of the elastic and viscoelastic parts of the energy density release}

The total energy density release $Y$ is written as the sum of the energy density releases associated to the single spring $Y_{e}$ and each Kelvin-Voigt 
branch $Y_{v_{i}}$ as expressed in (11). They can be formulated either in term of strain:

$$
\begin{gathered}
-Y_{e}=\rho \frac{\partial \psi_{e}}{\partial D}=-\frac{1}{2}\left(\boldsymbol{\varepsilon}-\sum_{i=1}^{N} \boldsymbol{\varepsilon}_{\boldsymbol{v}_{i}}-\boldsymbol{\varepsilon}_{\boldsymbol{p}}\right): \mathbb{C}_{\boldsymbol{e}}:\left(\boldsymbol{\varepsilon}-\sum_{i=1}^{N} \boldsymbol{\varepsilon}_{\boldsymbol{v}_{i}}-\boldsymbol{\varepsilon}_{\boldsymbol{p}}\right) \\
-Y_{v_{i}}=\rho \frac{\partial \psi_{v_{i}}}{\partial D}=-\frac{1}{2} \boldsymbol{\varepsilon}_{\boldsymbol{v}_{i}}: \mathbb{C}_{\boldsymbol{v}_{i}}: \boldsymbol{\varepsilon}_{\boldsymbol{v}_{i}}
\end{gathered}
$$

or, by introducing (7) into (A.1) and (8) into (A.2), in term of stress:

$$
\begin{gathered}
-Y_{e}=-\frac{e q(\boldsymbol{\sigma})^{2}}{2 E_{e}(1-D)^{2}} T_{e}, \\
-Y_{v_{i}}=-\frac{e q\left(\boldsymbol{\sigma}-\boldsymbol{\sigma}_{\boldsymbol{v}_{i}}\right)^{2}}{2 E_{v_{i}}(1-D)^{2}} T_{v_{i}},
\end{gathered}
$$

where $T_{e}$ ans $T_{v_{i}}$ are the stress triaxiality functions of the single spring and the $i^{\text {th }}$ Kelvin-Voigt branch, respectively:

$$
\begin{gathered}
T_{e}=\frac{2}{3}(1+\nu)+3(1-2 \nu)\left[\frac{h y d(\boldsymbol{\sigma})}{e q(\boldsymbol{\sigma})}\right]^{2}, \\
T_{v_{i}}=\frac{2}{3}(1+\nu)+3(1-2 \nu)\left[\frac{h y d\left(\boldsymbol{\sigma}-\boldsymbol{\sigma}_{\boldsymbol{v}_{i}}\right)}{e q\left(\boldsymbol{\sigma}-\boldsymbol{\sigma}_{\boldsymbol{v}_{i}}\right)}\right]^{2} .
\end{gathered}
$$

\section{Appendix B. Linearisation of the constitutive equations with the "convex cutting plane" method}

It is reminded that, when using the "convex cutting plane" form of the "return mapping algorithm" (Ortiz and Simo, 1986; Simo and Hughes, 1998), a simplification regarding the linearisation of the flow equations is made. Indeed, the gradients of the flows are not considered. Thus, with the proposed model, the linearisation of the flow equations (28) are simply given by:

$$
\delta \varepsilon_{\boldsymbol{p}}=\frac{\boldsymbol{\Lambda}(\boldsymbol{\sigma})}{1-D} \delta r, \quad \delta D=\frac{\Omega(Y)}{1-D} \delta r
$$


With this in mind, the linearisation of the residuals (32 and 33) and the stress (7) are expressed as follow:

$$
\begin{aligned}
& \delta \boldsymbol{\phi}_{\boldsymbol{v}_{i}}=\frac{\partial \boldsymbol{\phi}_{\boldsymbol{v}_{i}}}{\partial \boldsymbol{\sigma}}: \delta \boldsymbol{\sigma}+\frac{\partial \boldsymbol{\phi}_{\boldsymbol{v}_{i}}}{\partial \boldsymbol{\varepsilon}_{\boldsymbol{v}_{i}}}: \delta \boldsymbol{\varepsilon}_{\boldsymbol{v}_{i}}+\frac{\partial \boldsymbol{\phi}_{\boldsymbol{v}_{i}}}{\partial \dot{\boldsymbol{\varepsilon}}_{\boldsymbol{v}_{i}}}: \delta \dot{\boldsymbol{\varepsilon}}_{\boldsymbol{v}_{i}}+\frac{\partial \phi_{\boldsymbol{v}_{i}}}{\partial D} \delta D \\
& =\frac{\partial \boldsymbol{\phi}_{\boldsymbol{v}_{i}}}{\partial \boldsymbol{\sigma}}: \delta \boldsymbol{\sigma}+\frac{\partial \boldsymbol{\phi}_{\boldsymbol{v}_{i}}}{\partial \boldsymbol{\varepsilon}_{\boldsymbol{v}_{i}}}: \delta \boldsymbol{\varepsilon}_{\boldsymbol{v}_{i}}+\frac{\partial \boldsymbol{\phi}_{\boldsymbol{v}_{i}}}{\partial \dot{\boldsymbol{\varepsilon}}_{\boldsymbol{v}_{i}}} \frac{1}{\Delta t}: \delta \boldsymbol{\varepsilon}_{\boldsymbol{v}_{i}}+\frac{\partial \boldsymbol{\phi}_{\boldsymbol{v}_{i}}}{\partial D} \frac{\Omega(Y)}{1-D} \delta r \\
& =\frac{\partial \boldsymbol{\phi}_{\boldsymbol{v}_{i}}}{\partial \boldsymbol{\sigma}}: \delta \boldsymbol{\sigma}+\left(\frac{\partial \boldsymbol{\phi}_{\boldsymbol{v}_{i}}}{\partial \boldsymbol{\varepsilon}_{\boldsymbol{v}_{i}}}+\frac{\partial \boldsymbol{\phi}_{\boldsymbol{v}_{i}}}{\partial \dot{\boldsymbol{\varepsilon}}_{\boldsymbol{v}_{i}}} \frac{1}{\Delta t}\right): \delta \boldsymbol{\varepsilon}_{\boldsymbol{v}_{i}}+\frac{\partial \boldsymbol{\phi}_{\boldsymbol{v}_{i}}}{\partial D} \frac{\Omega(Y)}{1-D} \delta r \\
& =\mathbb{A}_{\boldsymbol{v}_{i} \boldsymbol{\sigma}}: \delta \boldsymbol{\sigma}+\mathbb{A}_{\boldsymbol{v}_{i} \boldsymbol{v}_{i}}: \delta \boldsymbol{\varepsilon}_{\boldsymbol{v}_{i}}+\boldsymbol{A}_{\boldsymbol{v}_{i} \boldsymbol{r}} \delta r, \\
& \delta \phi_{r}=\frac{\partial \phi_{r}}{\partial \boldsymbol{\sigma}}: \delta \boldsymbol{\sigma}+\frac{\partial \phi_{r}}{\partial r} \delta r+\frac{\partial \phi_{r}}{\partial \dot{r}} \delta \dot{r}+\frac{\partial \phi_{r}}{\partial D} \delta D \\
& =\frac{\partial \phi_{r}}{\partial \boldsymbol{\sigma}}: \delta \boldsymbol{\sigma}+\frac{\partial \phi_{r}}{\partial r} \delta r+\frac{\partial \phi_{r}}{\partial \dot{r}} \frac{1}{\Delta t} \delta r+\frac{\partial \phi_{r}}{\partial D} \frac{\Omega(Y)}{1-D} \delta r \\
& =\frac{\partial \phi_{r}}{\partial \boldsymbol{\sigma}}: \delta \boldsymbol{\sigma}+\left(\frac{\partial \phi_{r}}{\partial r}+\frac{\partial \phi_{r}}{\partial \dot{r}} \frac{1}{\Delta t}+\frac{\partial \phi_{r}}{\partial D} \frac{\Omega(Y)}{1-D}\right) \delta r \\
& =\boldsymbol{A}_{\boldsymbol{r} \boldsymbol{\sigma}}: \delta \boldsymbol{\sigma}+A_{r r} \delta r \\
& \delta \boldsymbol{\sigma}=\frac{\partial \boldsymbol{\sigma}}{\partial \boldsymbol{\varepsilon}}: \delta \boldsymbol{\varepsilon}+\sum_{j=1}^{N} \frac{\partial \boldsymbol{\sigma}}{\partial \boldsymbol{\varepsilon}_{\boldsymbol{v}_{j}}}: \delta \boldsymbol{\varepsilon}_{\boldsymbol{v}_{j}}+\frac{\partial \boldsymbol{\sigma}}{\partial \boldsymbol{\varepsilon}_{\boldsymbol{p}}}: \delta \boldsymbol{\varepsilon}_{\boldsymbol{p}}+\frac{\partial \boldsymbol{\sigma}}{\partial D} \delta D \\
& =\frac{\partial \boldsymbol{\sigma}}{\partial \boldsymbol{\varepsilon}}: \delta \boldsymbol{\varepsilon}+\sum_{j=1}^{N} \frac{\partial \boldsymbol{\sigma}}{\partial \boldsymbol{\varepsilon}_{\boldsymbol{v}_{j}}}: \delta \boldsymbol{\varepsilon}_{\boldsymbol{v}_{j}}+\frac{\partial \boldsymbol{\sigma}}{\partial \boldsymbol{\varepsilon}_{\boldsymbol{p}}}: \frac{\boldsymbol{\Lambda}(\boldsymbol{\sigma})}{1-D} \delta r+\frac{\partial \boldsymbol{\sigma}}{\partial D} \frac{\Omega(Y)}{1-D} \delta r \\
& =\frac{\partial \boldsymbol{\sigma}}{\partial \boldsymbol{\varepsilon}}: \delta \boldsymbol{\varepsilon}+\sum_{j=1}^{N} \frac{\partial \boldsymbol{\sigma}}{\partial \boldsymbol{\varepsilon}_{\boldsymbol{v}_{j}}}: \delta \boldsymbol{\varepsilon}_{\boldsymbol{v}_{j}}+\left(\frac{\partial \boldsymbol{\sigma}}{\partial \boldsymbol{\varepsilon}_{\boldsymbol{p}}}: \frac{\boldsymbol{\Lambda}(\boldsymbol{\sigma})}{1-D}+\frac{\partial \boldsymbol{\sigma}}{\partial D} \frac{\Omega(Y)}{1-D}\right) \delta r \\
& =\mathbb{B}_{\boldsymbol{\sigma} \varepsilon}: \delta \varepsilon+\sum_{j=1}^{N} \mathbb{B}_{\boldsymbol{\sigma} \boldsymbol{v}_{j}}: \delta \boldsymbol{\varepsilon}_{\boldsymbol{v}_{j}}+\boldsymbol{B}_{\boldsymbol{\sigma} \boldsymbol{r}} \delta r .
\end{aligned}
$$

with

$$
\begin{gathered}
\mathbb{A}_{\boldsymbol{v}_{i} \boldsymbol{\sigma}}=\frac{\partial \boldsymbol{\phi}_{\boldsymbol{v}_{i}}}{\partial \boldsymbol{\sigma}}=-\frac{\mathbb{V}_{\boldsymbol{v}_{i}}^{-1}}{1-D} \\
\mathbb{A}_{\boldsymbol{v}_{i} \boldsymbol{v}_{i}}=\frac{\partial \boldsymbol{\phi}_{\boldsymbol{v}_{i}}}{\partial \boldsymbol{\varepsilon}_{\boldsymbol{v}_{i}}}+\frac{\partial \boldsymbol{\phi}_{\boldsymbol{v}_{i}}}{\partial \dot{\boldsymbol{\varepsilon}}_{\boldsymbol{v}_{i}}} \frac{1}{\Delta t}=\mathbb{V}_{\boldsymbol{v}_{i}}^{-1}: \mathbb{C}_{\boldsymbol{v}_{i}}+\frac{\mathbb{I}}{\Delta t}
\end{gathered}
$$




$$
\begin{gathered}
\boldsymbol{A}_{\boldsymbol{v}_{i} \boldsymbol{r}}=\frac{\partial \boldsymbol{\phi}_{\boldsymbol{v}_{i}}}{\partial D} \frac{\Omega(Y)}{1-D}=-\frac{\mathbb{V}_{\boldsymbol{v}_{i}}^{-1}: \boldsymbol{\sigma} \Omega(Y)}{(1-D)^{3}} \\
\boldsymbol{A}_{\boldsymbol{r} \boldsymbol{\sigma}}=\frac{\partial \phi_{r}}{\partial \boldsymbol{\sigma}}=-\frac{\partial Q^{-1}}{\partial f} \frac{\boldsymbol{\Lambda}(\boldsymbol{\sigma})}{1-D} \\
A_{r r}=\frac{\partial \phi_{r}}{\partial r}+\frac{\partial \phi_{r}}{\partial \dot{r}} \frac{1}{\Delta t}+\frac{\partial \phi_{r}}{\partial D} \frac{\Omega(Y)}{1-D}=\frac{\partial Q^{-1}}{\partial f}\left(\frac{\partial R}{\partial r}-\frac{e q(\boldsymbol{\sigma}) \Omega(Y)}{(1-D)^{3}}\right)+\frac{1}{\Delta t} . \\
\mathbb{B}_{\boldsymbol{\sigma \varepsilon}}=\frac{\partial \boldsymbol{\sigma}}{\partial \boldsymbol{\varepsilon}}=(1-D) \mathbb{C}_{\boldsymbol{e}} \\
\mathbb{B}_{\boldsymbol{\sigma} \boldsymbol{v}_{j}}=\frac{\partial \boldsymbol{\sigma}}{\partial \boldsymbol{\varepsilon}_{\boldsymbol{v}_{j}}}=-(1-D) \mathbb{C}_{\boldsymbol{e}} \\
\boldsymbol{B}_{\boldsymbol{\sigma} \boldsymbol{r}}=\frac{\partial \boldsymbol{\sigma}}{\partial \boldsymbol{\varepsilon}_{\boldsymbol{p}}}: \frac{\boldsymbol{\Lambda}(\boldsymbol{\sigma})}{1-D}+\frac{\partial \boldsymbol{\sigma}}{\partial D} \frac{\Omega(Y)}{1-D}=-\mathbb{C}_{\boldsymbol{e}}:\left[\frac{\left(\boldsymbol{\varepsilon}-\sum_{i=1}^{N} \boldsymbol{\varepsilon}_{\boldsymbol{v}_{i}}-\boldsymbol{\varepsilon}_{\boldsymbol{p}}\right) \Omega(Y)}{1-D}+\boldsymbol{\Lambda}(\boldsymbol{\sigma})\right]
\end{gathered}
$$

\section{Appendix C. Corrector systems}

The "viscoelastic corrector system" is built by introducing (B.4) into (B.2) while considering $\delta \boldsymbol{\varepsilon}=0, \delta r=0, \delta \boldsymbol{\varepsilon}_{\boldsymbol{p}}=0, \delta D=0$ and $\boldsymbol{\phi}_{\boldsymbol{v}_{i}}+\delta \boldsymbol{\phi}_{\boldsymbol{v}_{i}}=0$. Its purpose is to establish a linear relationship between $\delta \varepsilon_{\boldsymbol{v}_{\boldsymbol{i}}}$ and $\boldsymbol{\phi}_{\boldsymbol{v}_{\boldsymbol{i}}}$ such it is mentioned in (37). Its expression is given by:

$$
\left(\begin{array}{ccccc}
\mathbb{L}_{\boldsymbol{v}_{1} \boldsymbol{v}_{1}}^{*} & \mathbb{L}_{\boldsymbol{v}_{1} \boldsymbol{v}_{2}}^{*} & \mathbb{L}_{\boldsymbol{v}_{1} \boldsymbol{v}_{3}}^{*} & \ldots & \mathbb{L}_{\boldsymbol{v}_{1} \boldsymbol{v}_{N}}^{*} \\
\mathbb{L}_{\boldsymbol{v}_{2} \boldsymbol{v}_{1}}^{*} & \mathbb{L}_{\boldsymbol{v}_{2} \boldsymbol{v}_{2}}^{*} & \mathbb{L}_{\boldsymbol{v}_{2} \boldsymbol{v}_{3}}^{*} & \ldots & \mathbb{L}_{\boldsymbol{v}_{2} \boldsymbol{v}_{N}}^{*} \\
\mathbb{L}_{\boldsymbol{v}_{3} \boldsymbol{v}_{1}}^{*} & \mathbb{L}_{\boldsymbol{v}_{3} \boldsymbol{v}_{2}}^{*} & \mathbb{L}_{\boldsymbol{v}_{3} \boldsymbol{v}_{3}}^{*} & \ldots & \mathbb{L}_{\boldsymbol{v}_{3} \boldsymbol{v}_{N}}^{*} \\
\vdots & \vdots & \vdots & \ddots & \vdots \\
\mathbb{L}_{\boldsymbol{v}_{N} \boldsymbol{v}_{1}}^{*} & \mathbb{L}_{\boldsymbol{v}_{N} \boldsymbol{v}_{2}}^{*} & \mathbb{L}_{\boldsymbol{v}_{N} \boldsymbol{v}_{3}}^{*} & \ldots & \mathbb{L}_{\boldsymbol{v}_{N} \boldsymbol{v}_{N}}^{*}
\end{array}\right)=\left(\begin{array}{ccccc}
\mathbb{K}_{\boldsymbol{v}_{1} \boldsymbol{v}_{1}} & \mathbb{K}_{\boldsymbol{v}_{1} \boldsymbol{v}_{2}} & \mathbb{K}_{\boldsymbol{v}_{1} \boldsymbol{v}_{3}} & \ldots & \mathbb{K}_{\boldsymbol{v}_{1} \boldsymbol{v}_{N}} \\
\mathbb{K}_{\boldsymbol{v}_{2} \boldsymbol{v}_{1}} & \mathbb{K}_{\boldsymbol{v}_{2} \boldsymbol{v}_{2}} & \mathbb{K}_{\boldsymbol{v}_{2} \boldsymbol{v}_{3}} & \ldots & \mathbb{K}_{\boldsymbol{v}_{2} \boldsymbol{v}_{N}} \\
\mathbb{K}_{\boldsymbol{v}_{3} \boldsymbol{v}_{1}} & \mathbb{K}_{\boldsymbol{v}_{3} \boldsymbol{v}_{2}} & \mathbb{K}_{\boldsymbol{v}_{3} \boldsymbol{v}_{3}} & \ldots & \mathbb{K}_{\boldsymbol{v}_{3} \boldsymbol{v}_{N}} \\
\vdots & \vdots & \vdots & \ddots & \vdots \\
\mathbb{K}_{\boldsymbol{v}_{N} \boldsymbol{v}_{1}} & \mathbb{K}_{\boldsymbol{v}_{N} \boldsymbol{v}_{2}} & \mathbb{K}_{\boldsymbol{v}_{N} \boldsymbol{v}_{3}} & \ldots & \mathbb{K}_{\boldsymbol{v}_{N} \boldsymbol{v}_{N}}
\end{array}\right)^{-1}
$$

The "full corrector system" is built by introducing (B.4) into (B.2) while considering only $\delta \varepsilon=0, \boldsymbol{\phi}_{\boldsymbol{v}_{i}}+\delta \boldsymbol{\phi}_{\boldsymbol{v}_{i}}=0$ and $\phi_{r}+\delta \phi_{r}=0$. Its purpose 
is to establish a linear relationship between $\delta \boldsymbol{\varepsilon}_{\boldsymbol{v}_{\boldsymbol{i}}}, \delta r, \boldsymbol{\phi}_{\boldsymbol{v}_{\boldsymbol{i}}}$ and $\phi_{r}$, such it is mentioned in (42). Its expression is given by:

$$
\left(\begin{array}{cccccc}
\mathbb{L}_{\boldsymbol{v}_{1} \boldsymbol{v}_{1}} & \mathbb{L}_{\boldsymbol{v}_{1} \boldsymbol{v}_{2}} & \mathbb{L}_{\boldsymbol{v}_{1} \boldsymbol{v}_{3}} & \ldots & \mathbb{L}_{\boldsymbol{v}_{1} \boldsymbol{v}_{N}} & \boldsymbol{L}_{\boldsymbol{v}_{1} \boldsymbol{r}} \\
\mathbb{L}_{\boldsymbol{v}_{2} \boldsymbol{v}_{1}} & \mathbb{L}_{\boldsymbol{v}_{2} \boldsymbol{v}_{2}} & \mathbb{L}_{\boldsymbol{v}_{2} \boldsymbol{v}_{3}} & \ldots & \mathbb{L}_{\boldsymbol{v}_{2} \boldsymbol{v}_{N}} & \boldsymbol{L}_{\boldsymbol{v}_{2} \boldsymbol{r}} \\
\mathbb{L}_{\boldsymbol{v}_{3} \boldsymbol{v}_{1}} & \mathbb{L}_{\boldsymbol{v}_{3} \boldsymbol{v}_{2}} & \mathbb{L}_{\boldsymbol{v}_{3} \boldsymbol{v}_{3}} & \ldots & \mathbb{L}_{\boldsymbol{v}_{3} \boldsymbol{v}_{N}} & \boldsymbol{L}_{\boldsymbol{v}_{3} \boldsymbol{r}} \\
\vdots & \vdots & \vdots & \ddots & \vdots & \vdots \\
\mathbb{L}_{\boldsymbol{v}_{N} \boldsymbol{v}_{1}} & \mathbb{L}_{\boldsymbol{v}_{N} \boldsymbol{v}_{2}} & \mathbb{L}_{\boldsymbol{v}_{N} \boldsymbol{v}_{3}} & \ldots & \mathbb{L}_{\boldsymbol{v}_{N} \boldsymbol{v}_{N}} & \boldsymbol{L}_{\boldsymbol{v}_{N} \boldsymbol{r}} \\
\boldsymbol{L}_{\boldsymbol{r} \boldsymbol{v}_{1}} & \boldsymbol{L}_{\boldsymbol{r} \boldsymbol{v}_{2}} & \boldsymbol{L}_{\boldsymbol{r} \boldsymbol{v}_{3}} & \ldots & \boldsymbol{L}_{\boldsymbol{r} \boldsymbol{v}_{N}} & L_{r r}
\end{array}\right)=\left(\begin{array}{cccccc}
\mathbb{K}_{\boldsymbol{v}_{1} \boldsymbol{v}_{1}} & \mathbb{K}_{\boldsymbol{v}_{1} \boldsymbol{v}_{2}} & \mathbb{K}_{\boldsymbol{v}_{1} \boldsymbol{v}_{3}} & \ldots & \mathbb{K}_{\boldsymbol{v}_{1} \boldsymbol{v}_{N}} & \boldsymbol{K}_{\boldsymbol{v}_{1} \boldsymbol{r}} \\
\mathbb{K}_{\boldsymbol{v}_{2} \boldsymbol{v}_{1}} & \mathbb{K}_{\boldsymbol{v}_{2} \boldsymbol{v}_{2}} & \mathbb{K}_{\boldsymbol{v}_{2} \boldsymbol{v}_{3}} & \ldots & \mathbb{K}_{\boldsymbol{v}_{2} \boldsymbol{v}_{N}} & \boldsymbol{K}_{\boldsymbol{v}_{2} \boldsymbol{r}} \\
\mathbb{K}_{\boldsymbol{v}_{3} \boldsymbol{v}_{1}} & \mathbb{K}_{\boldsymbol{v}_{3} \boldsymbol{v}_{2}} & \mathbb{K}_{\boldsymbol{v}_{3} \boldsymbol{v}_{3}} & \ldots & \mathbb{K}_{\boldsymbol{v}_{3} \boldsymbol{v}_{N}} & \boldsymbol{K}_{\boldsymbol{v}_{3} \boldsymbol{r}} \\
\vdots & \vdots & \vdots & \ddots & \vdots & \vdots \\
\mathbb{K}_{\boldsymbol{v}_{N} \boldsymbol{v}_{1}} & \mathbb{K}_{\boldsymbol{v}_{N} \boldsymbol{v}_{2}} & \mathbb{K}_{\boldsymbol{v}_{N} \boldsymbol{v}_{3}} & \ldots & \mathbb{K}_{\boldsymbol{v}_{N} \boldsymbol{v}_{N}} & \boldsymbol{K}_{\boldsymbol{v}_{N} \boldsymbol{r}} \\
\boldsymbol{K}_{\boldsymbol{r} \boldsymbol{v}_{1}} & \boldsymbol{K}_{\boldsymbol{r} \boldsymbol{v}_{2}} & \boldsymbol{K}_{\boldsymbol{r} \boldsymbol{v}_{3}} & \ldots & \boldsymbol{K}_{\boldsymbol{r} \boldsymbol{v}_{N}} & K_{r r}
\end{array}\right)
$$

with

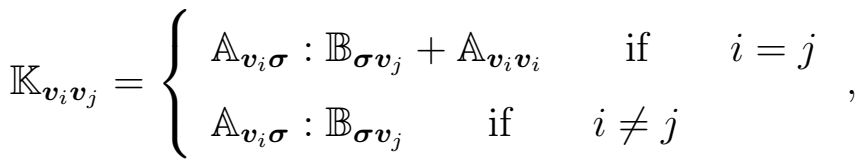

$$
\begin{aligned}
& \boldsymbol{K}_{\boldsymbol{v}_{i} \boldsymbol{r}}=\mathbb{A}_{\boldsymbol{v}_{i} \boldsymbol{\sigma}}: \boldsymbol{B}_{\boldsymbol{\sigma} \boldsymbol{r}}+\boldsymbol{A}_{\boldsymbol{v}_{i} \boldsymbol{r}}, \\
& \boldsymbol{K}_{\boldsymbol{r} \boldsymbol{v}_{j}}=\boldsymbol{A}_{\boldsymbol{r} \boldsymbol{\sigma}}: \mathbb{B}_{\boldsymbol{\sigma} \boldsymbol{v}_{j}}, \\
& K_{r r}=\boldsymbol{A}_{\boldsymbol{r} \boldsymbol{\sigma}}: \boldsymbol{B}_{\boldsymbol{\sigma} r}+A_{r r} .
\end{aligned}
$$

\section{Appendix D. Tangent multipliers}

The "tangent multipliers" $\mathbb{X}_{\boldsymbol{v}_{j} \varepsilon}^{*}$ are deduced by introducing (43) into (44). Then, a linear relationship can be identified between each $d \boldsymbol{\varepsilon}_{\boldsymbol{v}_{i}}$ and $d \boldsymbol{\varepsilon}$ :

$$
\begin{aligned}
d \boldsymbol{\phi}_{\boldsymbol{v}_{i}} & =\sum_{j=1}^{N} \mathbb{K}_{\boldsymbol{v}_{i} \boldsymbol{v}_{j}}: d \boldsymbol{\varepsilon}_{\boldsymbol{v}_{j}}+\mathbb{A}_{\boldsymbol{v}_{i} \boldsymbol{\sigma}}: \mathbb{B}_{\boldsymbol{\sigma} \boldsymbol{\varepsilon}}: d \boldsymbol{\varepsilon}=0 \\
& \mathbb{1} \\
d \boldsymbol{\varepsilon}_{\boldsymbol{v}_{j}} & =\left(-\sum_{i=1}^{N} \mathbb{L}_{\boldsymbol{v}_{j} \boldsymbol{v}_{i}}^{*}: \mathbb{A}_{\boldsymbol{v}_{i} \boldsymbol{\sigma}}: \mathbb{B}_{\boldsymbol{\sigma \varepsilon}}\right): d \boldsymbol{\varepsilon} \\
& =\mathbb{X}_{\boldsymbol{v}_{j} \varepsilon}^{*}: d \boldsymbol{\varepsilon}
\end{aligned}
$$


where

$$
\mathbb{X}_{\boldsymbol{v}_{j} \varepsilon}^{*}=-\sum_{i=1}^{N} \mathbb{L}_{\boldsymbol{v}_{j} \boldsymbol{v}_{i}}^{*}: \mathbb{A}_{\boldsymbol{v}_{i} \boldsymbol{\sigma}}: \mathbb{B}_{\boldsymbol{\sigma \varepsilon} \boldsymbol{\varepsilon}}
$$

The "tangent multipliers" $\mathbb{X}_{\boldsymbol{v}_{\boldsymbol{j}} \boldsymbol{\varepsilon}}$ and $\boldsymbol{X}_{\boldsymbol{r} \varepsilon}$ are deduced by introducing (47) into (48). Then, a linear relationship can be identified between each $d \boldsymbol{\varepsilon}_{\boldsymbol{v}_{i}}$ and $d \varepsilon$, and between $d r$ and $d \varepsilon$ :

$$
\begin{aligned}
& \left\{\begin{array}{c}
d \boldsymbol{\phi}_{\boldsymbol{v}_{i}}=\sum_{j=1}^{N} \mathbb{K}_{\boldsymbol{v}_{i} \boldsymbol{v}_{j}}: d \boldsymbol{\varepsilon}_{\boldsymbol{v}_{j}}+\boldsymbol{K}_{\boldsymbol{v}_{i} \boldsymbol{r}} d r+\mathbb{A}_{\boldsymbol{v}_{i} \boldsymbol{\sigma}}: \mathbb{B}_{\boldsymbol{\sigma} \boldsymbol{\varepsilon}}: d \boldsymbol{\varepsilon}=0 \\
d \phi_{r}=\sum_{j=1}^{N} \boldsymbol{K}_{\boldsymbol{r} \boldsymbol{v}_{j}}: d \boldsymbol{\varepsilon}_{\boldsymbol{v}_{j}}+K_{r r} d r+\boldsymbol{A}_{\boldsymbol{r} \boldsymbol{\sigma}}: \mathbb{B}_{\boldsymbol{\sigma} \boldsymbol{\varepsilon}}: d \boldsymbol{\varepsilon}=0
\end{array}\right. \\
& \mathbb{1} \\
& \left\{\begin{array}{rl}
d \boldsymbol{\varepsilon}_{\boldsymbol{v}_{j}} & =\left(-\sum_{i=1}^{N} \mathbb{L}_{\boldsymbol{v}_{j} \boldsymbol{v}_{i}}: \mathbb{A}_{\boldsymbol{v}_{i} \boldsymbol{\sigma}}: \mathbb{B}_{\boldsymbol{\sigma} \boldsymbol{\varepsilon}}-\boldsymbol{L}_{\boldsymbol{v}_{j} \boldsymbol{r}} \otimes \boldsymbol{A}_{\boldsymbol{r} \boldsymbol{\sigma}}: \mathbb{B}_{\boldsymbol{\sigma} \boldsymbol{\varepsilon}}\right): d \boldsymbol{\varepsilon} \\
& =\mathbb{X}_{\boldsymbol{v}_{j} \boldsymbol{\varepsilon}}: d \boldsymbol{\varepsilon} \\
d r & =\left(-\sum_{i=1}^{N} \boldsymbol{L}_{\boldsymbol{r} \boldsymbol{v}_{i}}: \mathbb{A}_{\boldsymbol{v}_{i} \boldsymbol{\sigma}}: \mathbb{B}_{\boldsymbol{\sigma} \boldsymbol{\varepsilon}}-L_{r r} \boldsymbol{A}_{\boldsymbol{r} \boldsymbol{\sigma}}: \mathbb{B}_{\boldsymbol{\sigma} \boldsymbol{\varepsilon}}\right): d \boldsymbol{\varepsilon} \\
& =\boldsymbol{X}_{\boldsymbol{r} \boldsymbol{\varepsilon}}: d \boldsymbol{\varepsilon}
\end{array},\right.
\end{aligned}
$$

where

$$
\begin{gathered}
\mathbb{X}_{\boldsymbol{v}_{j} \varepsilon}=-\sum_{i=1}^{N} \mathbb{L}_{\boldsymbol{v}_{j} \boldsymbol{v}_{i}}: \mathbb{A}_{\boldsymbol{v}_{i} \boldsymbol{\sigma}}: \mathbb{B}_{\boldsymbol{\sigma} \boldsymbol{\varepsilon}}-\boldsymbol{L}_{\boldsymbol{v}_{j} \boldsymbol{r}} \otimes \boldsymbol{A}_{\boldsymbol{r} \boldsymbol{\sigma}}: \mathbb{B}_{\boldsymbol{\sigma} \varepsilon} \\
\boldsymbol{X}_{\boldsymbol{r} \varepsilon}=-\sum_{i=1}^{N} \boldsymbol{L}_{\boldsymbol{r} \boldsymbol{v}_{i}}: \mathbb{A}_{\boldsymbol{v}_{i} \boldsymbol{\sigma}}: \mathbb{B}_{\boldsymbol{\sigma} \varepsilon}-L_{r r} \boldsymbol{A}_{\boldsymbol{r} \boldsymbol{\sigma}}: \mathbb{B}_{\boldsymbol{\sigma} \boldsymbol{\varepsilon}}
\end{gathered}
$$

\section{References}

Achour, N., Chatzigeorgiou, G., Meraghni, F., Chemisky, Y., Fitoussi, J., 2015. Implicit implementation and consistent tangent modulus of a viscoplastic model for polymers. International Journal of Mechanical Sciences 103, 297-305. 
Arif, M.-F., Meraghni, F., Chemisky, Y., Despringe, N., Robert, G., 2014 a. In situ damage mechanisms investigation of PA66/GF30 composite: Effect of relative humidity. Composites: Part B 58, 487-495.

Arif, M.-F., Saintier, N., Meraghni, F., Fitoussi, J., Chemisky, Y., Robert, G., 2014b. Multiscale fatigue damage characterization in short glass fiber reinforced polyamide-66. Composites: Part B 61, 55-65.

Arruda, E.-M., Boyce, M.-C., Jayachandran, R., 1995. Effects of strain rate, temperature and thermomechanical coupling on the finite strain deformation of glassy polymers. Mechanics of Materials 19, 193-212.

Benaarbia, A., Chrysochoos, A., Robert, G., 2014. Kinetics of stored and dissipated energies associated with cyclic loadings of dry polyamide 6.6 specimens. polymer Testing 34, 155-167.

Benaarbia, A., Chrysochoos, A., Robert, G., 2015. Thermomechanical behavior of PA6.6 composites subjected to low cycle fatigue. Composites: Part B 76, 52-64.

Chaboche, J.-L., Kanouté, P., Roos, A., 2005. On the capabilities of meanfield approaches for the description of plasticity in metal matrix composites. International Journal of Plasticity 21, 1409-1434.

Chatzigeorgiou, G., Charalambakis, N., Chemisky, Y., Meraghni, F., 2016. Periodic homogenization for fully coupled thermomechanical modeling of dissipative generalized standard materials. International Journal of Plasticity $81,18-39$. 
Chatzigeorgiou, G., Chemisky, Y., Meraghni, F., 2015. Computational micro to macro transitions for shape memory alloy composites using periodic homogenization. Smart Materials and Structures 24.

Cojocaru, D., Karlsson, A., 2006. A simple numerical method of cycle jumps for cyclically loaded structures. International Journal of Fatigue 28, 16771689.

Detrez, F., Cantournet, S., Seguela, R., 2011. Plasticity/damage coupling in semi-crystalline polymers prior to yielding: Micromechanisms and damage law identification. Polymer 52, 1998-2008.

Drosdov, A.-D., Christiansen, J.-d., 2007. Cyclic viscoplasticity of solid polymers: The effects of strain rate and amplitude of deformation. Polymer 48, 3003-3012.

Fitoussi, J., Bocquet, M., Meraghni, F., 2013. Effect of the matrix behavior on the damage of ethylenepropylene glass fiber reinforced composite subjected to high strain rate tension. Composites: Part B 45, 1181-1191.

Gurson, A.-L., 1977. Continuum Theory of Ductile Rupture by Void Nucleation and Growth: Part I. Yield Criteria and Flow Rules for Porous Ductile Media. Journal of engineering materials and technology 99, 2-15.

Haouala, S., Doghri, I., 2015. Modeling and algorithms for two-scale time homogenizationof viscoelastic-viscoplastic solids under large numbers of cycles. International Journal of Plasticity 70, 98-125.

Khan, A.-S., Lopez-Pamies, O., Kazmi, R., 2006. Thermo-mechanical large deformation response and constitutive modeling of viscoelastic polymers 
over a wide range of strain rates and temperatures. International Journal of Plasticity 22, 581-601.

Krairi, A., Doghri, I., 2014. A thermodynamically-based constitutive model, for thermoplastic polymers coupling viscoelasticity viscoplasticity and ductile damage. International Journal of Plasticity 60, 163-181.

Lagoudas, D.-C., Gavazzi, A.-C., Nigam, H., 1991. Elastoplastic behavior of metal matrix composites based on incremental plasticity and the MoriTanaka averaging scheme. computational Mechanics 8, 193-203.

Lakes, R.-S., 1999. Viscoelastic Solids. CRC Press.

Launay, A., Maitournam, M.-H., Marco, Y., Raoult, I., 2013a. Multiaxial fatigue models for short glass fiber reinforced polyamide Part I: Nonlinear anisotropic constitutive behavior for cyclic response. International Journal of Fatigue 47, 382-389.

Launay, A., Maitournam, M.-H., Marco, Y., Raoult, I., 2013b. Multiaxial fatigue models for short glass fibre reinforced polyamide. Part II: Fatigue life estimation. International Journal of Fatigue 47, 2013.

Launay, A., Maitournam, M.-H., Marco, Y., Raoult, I., Szmytka, F., 2011. Cyclic behaviour of short glass fibre reinforced polyamide: Experimental study and constitutive equations. International Journal of Plasticity 27, 1267-1293.

Launay, A., Marco, Y., Maitournam, M.-H., Raoult, I., 2013c. Modelling the influence of temperature and relative humidity on the time-dependent me- 
chanical behaviour of a short glass fibre reinforced polyamide. Mechanics of Materials 56, 1-10.

Lawrimore II, W.-B., Francis, D.-K., Bouvard, J.-L., Hammi, Y., Horstemeyer, M.-F., 2016. A mesomechanics parametric finite element study of damage growth and coalescence in polymers using an ElastoviscoelasticViscoplastic internal state variable model. Mechanics of Materials 96, 8395.

Lemaitre, J., 1985. Coupled elasto-plasticity and damage constitutive equations. Computer methods in applied mechanics and engineering 51, 31-49.

Lemaitre, J., Chaboche, J.-L., 1990. Mechanics of solid materials. Cambridge University Press.

Lemaitre, J., Desmorat, R., 2005. Engineering damage mechanics : ductile, creep, fatigue and brittle failure. Springer.

Maurel-Pantel, A., Baquet, E., Bikard, J., Bouvard, J.-L., Billon, N., 2015. A thermo-mechanical large deformation constitutive model for polymers based on material network description: Application to a semi-crystalline polyamide 66. International Journal of Plasticity 67, 102-126.

Meraghni, F., Chemisky, Y., Piotrowski, B., Echchorfi, R., Bourgeois, N., Patoor, E., 2014. Parameter identification of a thermodynamic model for superelastic shape memory alloys using analytical calculation of the sensitivity matrix. European Journal of Mechanics - A/Solids 45, 226-237.

Meraghni, F., Nouri, H., Bourgeois, N., Czarnota, C., Lory, P., 2011. Parameters identification of fatigue damage model for short glass fiber reinforced 
polyamide (PA6-GF30) using digital image correlation. Procedia Engineering 10, 2110-2116.

Miled, B., Doghri, I., Brassart, L., Delannay, L., 2013. Micromechanical modeling of coupled viscoelasticviscoplastic composites based on an incrementally affine formulation. International Journal of Solids and Structures 50, 1755-1769.

Miled, B., Doghri, I., Delannay, L., 2011. Coupled viscoelasticviscoplastic modeling of homogeneous and isotropic polymers: Numerical algorithm and analytical solutions. Computer methods in applied mechanics and engineering 200, 3381-3394.

Moreau, S., Chrysochoos, A., Muracciole, J.-M., Wattrisse, B., 2005. Analysis of thermoelastic effects accompanying the deformation of PMMA and PC polymers. Comptes Rendus Mecanique 333, 648-653.

Nouri, H., Meraghni, F., Lory, P., 2009. Fatigue damage model for injectionmolded short glass fibre reinforced thermoplastics. International Journal of Fatigue 31, 934-942.

Ortiz, M., Simo, J.-C., 1986. An analysis of a new class of integration algorithms for elasto-plastic constitutive relations. International Journal for Numerical Methods in Engineering 23, 353-366.

Oshmyan, V.-G., Patlazhan, S.-A., Remond, Y., 2005. The Effect of Structural Changes and Nonlinear Character of Plastic Flow on Low Strains in Semicrystalline Polymers. Polymer Science 47, 346-351. 
Ottosen, N.-S., Ristinmaa, M., 2005. Introduction to time-dependent material behavior. In: The Mechanics of Constitutive Modeling. Elsevier Science, Ch. 14, pp. 357-378.

Ovalle Rodas, C., Zaïri, F., Naït-Abdelziz, M., 2014. A finite strain thermoviscoelastic constitutive model to describe the self-heating in elastomeric materials during low-cycle fatigue. Journal of the Mechanics and Physics of Solids 64, 396-410.

Ovalle Rodas, C., Zaïri, F., Naït-Abdelziz, M., Charrier, P., 2016. A thermovisco-hyperelastic model for the heat build-up during low-cycle fatigue of filled rubbers: Formulation, implementation and experimental verification. International Journal of Plasticity 79, 217-236.

Qidwai, M.-A., Lagoudas, D.-C., 2000. Numerical implementation of a shape memory alloy thermomechanical constitutive model using return mapping algorithms. International Journal for Numerical Methods in Engineering 47, 1123-1168.

Simo, J.-C., Hughes, T.-J.-R., 1998. Computational inelasticity. Springer.

Voyiadjis, G.-Z., Samadi-Dooki, A., 2016. Constitutive modeling of large inelastic deformation of amorphous polymers: Free volume and shear transformation zone dynamics. Journal of Applied Physics 119 (22), 225104.

Yu, C., Kang, G., Chen, K., 2017a. A hygro-thermo-mechanical coupled cyclic constitutive model for polymers with considering glass transition. International Journal of Plasticity 89, 29-65. 
Yu, C., Kang, G., Chen, K., Lu, F., 2017b. A thermo-mechanically coupled nonlinear viscoelasticviscoplastic cyclic constitutive model for polymeric materials. Mechanics of Materials 105, 1-15. 